\title{
An Improved Analytic Solution for Analysis of Particle Trajectories in Fibrous, Two-Dimensional Filters
}

\author{
H. Marshall \\ M. Sahraoui \\ M. Kaviany
}

For Subcontract \# B218839

Ending September 1993

September 1993

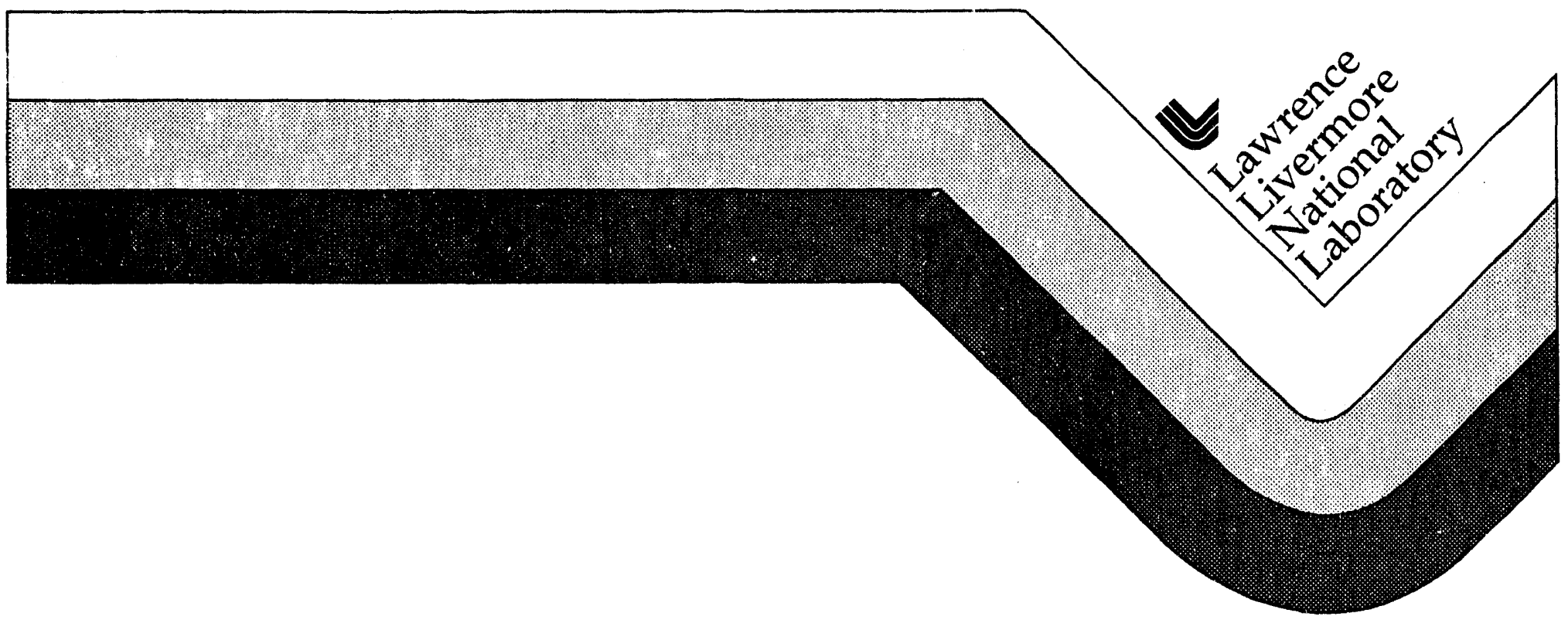




\section{DISCLAIMER}

Work performed under the auspices of the L.S. Department of Energy by Lawrence Livermore National Laboratory under contract number W-7405-ENG-48.

This document was prepared as an account of work sponsored by an agency of the United States Government. Neither the United States Government nor the University of California nor any of their employees, makes any warranty. express or implied, or assumes any legal liability or responsibility for the accuracy, completeness, or usefulness of any information, apparatus, product, or process disclosed. or represents that its use would not infringe privately owned rights. Reference herein to any specific commercial products, process, or service by trade name, trademark, manufacturer, or otherwise, does not necessarily constitute or imply its endorsement, recommendation, or favoring by the United States Government or the University of California. The views and opinions of authors expressed herein do not necessarily state or reflect those of the United States Government or the University of California. and shall not be used for advertising or product endorsement purposes. 


\title{
An Improved Analytic Solution for Analysis of Particle
} Trajectories in Fibrous, Two-Dimensional Filters

\author{
H. Marshall \\ Scientific Computation Group \\ M. Sahraoui and M. Kaviany \\ Department of Mechanical Engineering and Applied Mechanics \\ The University of Michigan \\ Ann Arbor, Michigan 48109
}




\begin{abstract}
Absiract
The Kuwabara solution for creeping fluid flow through periodic arrangement of cylinders is widely used in analytic and numerical studies of fibrous filters. Numerical solutions have shown that the Kuwabara solution has systematic errors and when used for the particle trajectories in filters it results in some error in the predicted filter efficiency. The numerical solutions although accurate, preclude further analytic treatments and are not as compact and convenient to use as the Kuwabara solution. By re-examining the outer boundary conditions of the Kuwabara solution, we have derived a correction term to the Kuwabara solution to obtain an extended solution that is more accurate and improves prediction of the filter efficiency. By comparison with the numerical solutions, it is shown that the Kuwabara solution is the high porosity asymptote and that the extended solution has an improved porosity dependence.

We explain a rectification which can make particle collection less efficient for periodic, inline arrangements of fibers with particle diffusion or body force. This rectification also results in the alignment of particles with inertia (i.e., high Stokes number particles).
\end{abstract}




\section{Introduction}

Studies of fibrous filters commonly use the idealization of the creeping fluid flow through a periodic arrangement of cylinders, as shown in Fig. 1(a). The assumption of periodicity allows for the analysis of single fiber with the appropriate boundary conditions. There is no exact analytic, closed-form compact solution for this two-dimensional flow. The Kuwabara ${ }^{1}$ creeping fluid flow solution uses a cylindrical unit-cell to approximate the local flow through arrangements of cylinders. When used in periodic arrangements of cylinders such as an in-line and isotropic arrangement, the Kuwabara approximation to the square unit cells leads to errors, because the Kuwabara solution does not satisfy the periodic boundary conditions.

The Kuwabara solution is widely used for studies of flow through fibrous filters (e.g., Banks², Banks and Kurowski ${ }^{3}$, and Choo and $\mathrm{Tien}^{4}$ ). Despite this shortcoming with the periodic boundary conditions, many useful results have been derived from the application of this solution to particle capturing in fibrous filters. The Kuwabara solution is compact and can be calculated with only a few operations which is important for example in the Monte Carlo simulations which require repeated calculations. As will be shown, the Kuwabara solution is also valid as a high porosity asymptote.

Accurate numerical solutions of the flow through equally spaced (i.e., isotropic), periodic arrangement of cylinders has been given by Sangani and Acrivos ${ }^{5}$. Even though their method can be used to obtain high accuracy, there are still some advantages in having an analytic solution as evidenced by the continued use of the Kuwabara solution. The Kuwabara solution is widely used because it is simple and allows for analytic treatment of the particle capture. However, as mentioned above it assumes a circular unit-cell, and therefore, lacks the accuracy 
in simulating the flow field in a square unit-cell. Here improvement to the Kuwabara solution is sought by using an extension that will improve the solution for the square unit-cell. This extension of the Kuwabara solution uses a geometric perturbation in order to obtain the square unit cell from the circular unit-cell. In order to access the accuracy of this approarh, this extended analytic solution is compared with the numerical solutions of fluid the flow through the in-line arrangement of fibers shown in Fig. 1 (a). Then this improved analytic solution, the Kuwabara and the numerical solutions are used to calculate the particle trajectories and the particle capture efficiency. The capture mechanisms considered here are impaction and sieving. Capture by impaction occurs when the particle touches the fiber and then it is assumed that the particle adheres to the surface. Capture by sieving occurs when the particle diameter is larger than the clearance between the fibers. Hence sieving is geometrical and occurs only in the entrance region of the filter.

In Section 2 we introduce the numerical solution for isotropic and anisotropic, in-line arrangements of cylinders. In Section 3 the analytic correction terms are derived. In Section 4 the local velocity and the stream function solutions and the errors are examined. Section 5 shows the comparisons of the impaction efficiencies. Section 6 evaluates the derived and the numerical inertial collection efficiencies. Section 7 exarnines particle rectification effects found in this study.

\section{Numerical Solution}

Sahraoui and Kaviany ${ }^{6}$ solved the Navier Stokes equation for the fluid flow in in-line and staggered arrangements of cylinders. Here the same numerical method is used to solve for 
the Stokean flow through an in-line arrangement of fibers. The periodic arrangement of fibers can be represented by the unit cell shown in Fig. 1 (b). Due to the presence of the fiber within the square unit cell, the Cartesian coordinates are not accurate for mapping the fiber unless a very large number of grid points is used. Accurate results can be obtained by using a domain decomposition, where a cylindrical grid net is used near the fiber and a Cartesian grid net is used away from the fiber. Iteration for the solution is performed in both grid nets and a bilinear interpolation is used to communicate between them. In the case where multiple fibers are used, the same procedure is repeated for every fiber. More details about the domain decomposition can be found in Sahraoui and Kaviany ${ }^{6}$ and Prata and Sparrow The equations are nondimensionalized using the length scale $\ell$, the size linear dimension of an isotropic unit-cell. For anisotropic unit cells, we use the length scale $\ell=\sqrt{\ell_{x} \ell_{y}}$ where $\ell_{x}$ and $\ell_{y}$ are the dimensions of the unit cell in the $x$-and $y$-directions, respectively. The volume-averaged, $x$-component of the velocity (or Darcean velocity) is used as the velocity scale and is given by

$$
u_{D}=\langle u\rangle=\int_{-0.5}^{0.5} u \mathrm{~d} y
$$

The governing equations for the fluid flow in the Cartesian coordinates are

$$
\begin{aligned}
\frac{\partial u}{\partial x}+\frac{\partial v}{\partial y} & =0 \\
-\frac{\partial p}{\partial x}+\frac{\partial^{2} u}{\partial x^{2}}+\frac{\partial^{2} u}{\partial y^{2}} & =0 \\
-\frac{\partial p}{\partial y}+\frac{\partial^{2} v}{\partial x^{2}}+\frac{\partial^{2} v}{\partial y^{2}} & =0
\end{aligned}
$$

and in the cylindrical coordinates, the radial and tangential components of velocity $\left(v_{r}\right.$ and $\left.v_{\theta}\right)$ are given by

$$
\frac{\partial r v_{r}}{\partial r}+\frac{\partial v_{\theta}}{\partial \theta}=0
$$




$$
\begin{array}{r}
-\frac{\partial p}{\partial r}+\left[\frac{1}{r} \frac{\partial}{\partial r}\left(r \frac{\partial v_{r}}{\partial r}\right)+\frac{1}{r} \frac{\partial}{\partial \theta}\left(\frac{1}{r} \frac{\partial v_{r}}{\partial \theta}\right)\right]+S_{r}=0 \\
-\frac{1}{r} \frac{\partial p}{\partial \theta}+\left[\frac{1}{r} \frac{\partial}{\partial r}\left(r \frac{\partial v_{\theta}}{\partial r}\right)+\frac{1}{r} \frac{\partial}{\partial \theta}\left(\frac{1}{r} \frac{\partial v_{\theta}}{\partial \theta}\right)\right]+S_{\theta}=0
\end{array}
$$

where the source terms $S_{r}$ and $S_{\theta}$ are

$$
\begin{aligned}
& S_{r}=\left\{-2 \frac{v_{r}}{r^{2}}+\frac{2}{r^{2}} \frac{\partial v_{\theta}}{\partial \theta}+\frac{1}{r} \frac{\partial}{\partial r}\left(r \frac{\partial v_{r}}{\partial r}\right)+\frac{\partial}{\partial \theta}\left[\frac{\partial}{\partial r}\left(\frac{v_{\theta}}{r}\right)\right]\right\} \\
& S_{\theta}=\left(-\frac{v_{\theta}}{r^{2}}+\frac{2}{r^{2}} \frac{\partial v_{r}}{\partial \theta}\right)
\end{aligned}
$$

The above equations are solved by using the no-slip boundary condition on the surface of the fiber and the periodic boundary conditions at the boundaries of the unit-cell, as shown in Fig. 1 (b).

The momentum equations are solved using the finite-volume method and the pressure correction method, as suggested by Patankar $^{8}$. As will be shown, for low porosities the numerical solutions are more accurate than the Kuwabara solution for the fluid flow in periodic arrangements of cylinders. The numerical integration also allows examination of the flow field in the entrance region of the filter. In this region the condition of periodicity is not valid. For the study of the particle capture this entrance effect can be important. In Section 6.2 we examine the results of the numerical simulations of the flow in the entrance region for the in-line arrangement of cylinders. For these entrance simulations, we use three fibers aligned in the direction of the flow (i.e., $x$-direction). Since the fluid flow is not periodic at the inlet, then different boundary conditions are used. At the inlet (i.e., $x=0$ ), we use

$$
u=1 \quad, \quad v=0 \quad x=0
$$


At the exit (i.e., $x=L$ ) we use the periodic boundary condition

$$
u(L, y)=u(L-1, y) \quad, \quad v(L, y)=v(L-1, y) \quad x=L
$$

\subsection{Two-Dimensional Anisotropy}

In modeling the flow through fibrous filters, the in-line arrangements of cylinders are usually used. In these models, the periodic unit-cell is chosen to have the same length in the longitudinal (along the flow) and the transverse (orthogonal to the flow) directions. However, examination of the micrographs of fibrous filters shows that the fibers are very close in the longitudinal direction and far apart in the transverse direction. This anisotropy, among other geometrical factors, contributes to the discrepancy between the predicted and the experimental results for the pressure drop in fibrous filters. Using the two-dimensional numerical simulation, we examine the effect of this anisotropy on the pressure drop by varying the cell dimensions in the $x$ and $y$ directions, as shown in Fig. 2 (a). The same porosity and the average flow rate are maintained the same and the pressure drop over the same distance, which is the dimension

of the isotropic cell $\left(\sqrt{\ell_{x} \ell_{y}}=1\right)$, is determined. The results of these computations are given in Fig. $2(\mathrm{~b})$ and they show that as the transverse period $\left(\ell_{y}\right)$ increases, the pressure drop across the same distance $\ell$ decreases significantly. This result is consistent with the experimental results which give a pressure drop that is lower by about $60 \%$ than that for the square (i.e., isotropic) unit-cells (as reported by Liu and Rubow ${ }^{10}$ ). For $\ell_{y} / \ell_{x}=4\left(\ell_{y}=2\right.$ and $\ell_{x}=0.5$ ) the pressure drop is about $25 \%$ of the pressure drop for the isotropic structure. This shows that a significant portion of the pressure drop is due to flow restriction between the cylinders. This is also shown by decreasing $\ell_{y}$, which results in a significant pressure drop, as shown in Fig. 2 (b). 


\section{Analytic Solutions}

First, in Section 3.1 the Kuwabara solution for the Stokean flow in a cylindrical domain, as shown in Fig. 1(c), is reviewed. Then in Section 3.2 the extension to this solution for the square unit-cell, also shown in Fig. 1 (c), is developed. In Section 3.3, the extension to the anisotropic, periodic arrangements is developed.

\subsection{Kuwabara Solution}

As mentioned above, the Kuwabara solution ${ }^{1}$ approximates the periodic structure by using a cylindrical outer boundary. The governing equation for the stream function $\psi$ is given by

$$
\nabla^{4} \psi=0
$$

The boundary conditions at the surface of the fiber are

$$
v_{r}=v_{\theta}=0 \quad \text { at } \quad r=R
$$

where $v_{r}$ and $v_{\theta}$ are given by

$$
v_{r}=\frac{1}{r} \frac{\partial \psi}{\partial \theta} \quad \text { and } \quad v_{\theta}=-\frac{\partial \psi}{\partial r}
$$

For the outer boundary, Kuwabara uses

$$
v_{r}=\cos \theta \quad \text { and } \quad \omega=0 \quad \text { at } \quad r=R+\Delta R,
$$

where $\omega$ is the vorticity. The solution to Eq. (12) is

$$
\psi(r, \theta)=R\left[C_{1}\left(\frac{r}{R}\right)^{3}+C_{2} \frac{r}{R}+C_{3} \frac{R}{r}+C_{4} \frac{r}{R} \ln \left(\frac{r}{R}\right)\right] \sin \theta
$$


The coefficients $C_{1}$ and $C_{4}$ are found using the boundary conditions (13)-(15) and they are given by

$$
\begin{array}{r}
C_{4}=-\frac{4}{2 \ln \epsilon+3-4 \epsilon+\epsilon^{2}} \\
C_{1}=-\frac{\epsilon C_{4}}{4} \\
C_{3}=C_{1}+\frac{C_{4}}{2} \\
C_{2}=-C_{1}-C_{3}
\end{array}
$$

where $\epsilon$ is the porosity given by

$$
\epsilon=1-\pi R^{2}
$$

and for the circular unit-cell it is given by

$$
\epsilon=1-\left(\frac{R}{R+\Delta R}\right)^{2} .
$$

\subsection{Extended Analytic Solution}

The purpose of extending or improving the Kuwabara solution are three fold. It is practical to find new solution which is more accurate, but still compact and not of overwhelming complexity nor a slowly converging series such as the one suggested by Hasimoto ${ }^{11}$.

Since the Kuwabara solution is in much use, and as we shall show later, in the limit of high porosity it is a good approximation, the new analytic solution is obtained by adding a correction term to the Kuwabara solution. This additive correction form is possible because of the linear superposition of the eigenfunctions of the biharmonic operator.

From the boundary conditions for the Kuwabara solution in Eq. (15), we note that the only element of a periodic cell or even a square surrounding cell is that the outer radius can 
be chosen so that the solid fraction of this cylindrical region is the same as the square unit cell. This is indeed how the Kuwabara solution is used (e.g., Banks²). No other influence of the square unit cell is found in the Kuwabara solution. This greatly reduces the mathematical complexity since the eigenfunctions of the equations have a convenient form in the cylindrical coordinates, but reduces the accuracy of the solution away from the cylinder which in turn affects the accuracy of the prediction of the particle trajectories and collections.

In this extended analytic solution we attempt to correct for this problem. Due to the symmetry of the geometry and the flow considered, we can fold the domain into one quarter as shown in Fig. 1 (c). The boundary conditions we use along the cylinder surface are the same as the no-slip boundary in the Kuwabara solution. These are the appropriate boundary conditions as long as the mean free path of fluid molecules is much smaller than the fiber diameter. The equations to be solved for this creeping flow are

$$
\begin{aligned}
& \nabla^{2} \psi=\omega \\
& \nabla^{2} \omega=0 .
\end{aligned}
$$

The stream function solution, which satisfies these governing equations in a square unit cell and the no-slip boundary conditions in the cylindrical coordinates, is known (e.g., Sangani and Acrivos $^{5}$ ) and is

$$
\begin{gathered}
\psi=\left\{a_{1} r^{3}\left[1-\frac{4 \ln r}{2 \ln R+1}\left(\frac{R}{r}\right)^{2} \frac{2 \ln R-1}{2 \ln R+1}\left(\frac{R}{r}\right)^{4}\right]+b_{1} R^{2} r\left[1-\frac{2 \ln r}{2 \ln R+1}-\left(\frac{R}{r}\right)^{2} \frac{1}{2 \ln R+1}\right]\right\} \\
\sin \theta+\sum_{n=2}^{N}\left\{a_{n} r^{2 n+1}\left[1-2 n\left(\frac{R}{r}\right)^{4 n-2}+(2 n-1)\left(\frac{R}{r}\right)^{4 n}\right]+\right.
\end{gathered}
$$




$$
\left.b_{n} a^{2} r^{2 n-1}\left[1(2 n-1)\left(\frac{R}{r}\right)^{4 n-4}+2(n-1)\left(\frac{R}{r}\right)^{4 n-2}\right]\right\} \sin (2 n-1) \theta
$$

Sangan: and Acrivos ${ }^{5}$ have used the collocation method (a spectral numeric method) to solve for the spectral coefficients $a_{n}$ and $b_{n}$. This is done by using the appropriate boundary conditions at 'some discrete points on the Cartesian boundary of the square unit cell. Then they form linear equations for the coefficients $a_{n}$ and $b_{n}$ for each boundary collocation point. This provides an accurate sumerical method of solving for the fluid flow.

The outer boundaries of the square unit-cell in Fig. 1 (c) can be represented, in the cylindrical coordinates, as having a distance $r$ from the center point and an angle $\theta$. Then we can define $\delta(\theta)$ such that when it is added to the outer radius $R+\Delta R$, it gives the square unit cell in the polar coordinates as

$$
r=(R+\Delta R)+\delta(\theta)
$$

Note that $\delta(\theta)$ for the square geometry is always less than 0.23 . Thus $\delta(\theta)$ is always a small parameter and can be considered as a perturbation. This is a perturbation expansion from a cylinder to a square. The expansion of the stream function is given by

$$
\psi=\psi_{0}+\delta \psi_{1}+\delta^{2} \psi_{2}
$$

Substituting this into the linear biharmonic Eq. (12) gives

$$
\begin{aligned}
& \nabla^{4} \psi_{0}=0 \quad \text { at order } \delta^{0} \\
& \nabla^{4} \psi_{1}=0 \quad \text { at order } \delta^{1} .
\end{aligned}
$$

The boundary conditions we will use are

$$
\frac{\partial v}{\partial x}=0 \text { at } x=0.5
$$


and

$$
\frac{\partial \omega}{\partial x}=0 \text { at } y=0.5
$$

The problem with these boundary conditions is that there is no explicit dependence on $\delta(\theta)$. This can be obtained by a perturbative expansion of the boundary condition and a Taylor expansion about $R+\Delta R$. The result is

$$
\frac{\partial v_{0}}{\partial x}(r, \theta)=\left.\frac{\partial v_{0}}{\partial x}(r, \theta)\right|_{R+\Delta R}+\left.\frac{\partial}{\partial r} \frac{\partial v_{0}}{\partial x}(r, \theta)\right|_{R+\Delta R} \delta(\theta)
$$

The order $\delta^{0}$ boundary condition is

$$
\left.\frac{\partial v_{0}}{\partial x}(r, \theta)\right|_{R+\Delta R}=0
$$

and for the $\delta^{1}$ is

$$
\left.\frac{\partial v_{1}}{\partial x}(r, \theta)\right|_{R+\Delta R}=\left.\frac{\partial v_{0}}{\partial x}(r, \theta)\right|_{R+\Delta R} \delta(\theta) .
$$

Note the explicit dependence of the boundary conditiol on the geometry. Then $\delta^{1}$ can be expanded in a Fourier series in $\sin [(2 n-1) \theta]$ and the boundary conditions are used to calculate the coefficients in Eq. (25).

The resulting solution is not very accurate for low truncations. For high truncations the formula becomes excessively large. Sangani and Acrivos ${ }^{5}$ used 6 to 40 terms for accurate numerical solutions. This analytic method will produce accurate results if enough terms are included from the expansions. However, it does not provide a useful, compact formula. For this analytic form, each successive coefficient depends on the previous coefficients. Thus the length of the analytic solution becomes overwhelming for this method. In contrast our goal is to obtain a reasonably compact analytic solution. 
The problem is that the basic state of the perturbation is not a very efficient approximation. The Kuwabara solution is a better approximation than the lowest term in Eq. (25) with the above approximations. The above solution method is more accurate only when enough expansion terms are included. Since our goal is to have both an accurate and compact formula, the Kuwabara solution is used as our basic state. Note that the stream function obtained by Kuwabara is essentially the $\sin \theta$ term in Eq. (25) with a particular choice of the coefficients. With the Kuwabara solution as the basic state, we proceed with a stream function given by

$$
\psi=\psi_{K}+\psi_{E}
$$

where $\psi_{K}$ is the stream function obtained from the Kuwabara solution $\psi_{E}$ is the extension which is that part of Eq. (25) having the higher order terms of $\sin [2(n-1) \theta]$. The advantage of this formulation is that the Kuwabara solution is a more efficient choice than the appropriate boundary conditions applied to the first term of Eq. (25). The problem with this formulation is that Kuwabara's boundary conditions are not appropriate to the square unit-cell. In order that 1. the total stream function, have the correct boundary conditions we must impose a boundary condition on $\psi$ and then subtract the Kuwabara solution to obtain the boundary condition for $\psi_{E}$. The two boundary conditions on the total stream function we use are

$$
\begin{aligned}
& \int_{0}^{1 / 2} u \mathrm{~d} y=\Delta \psi \\
& \int_{0}^{1 / 2} v \mathrm{~d} y=0
\end{aligned}
$$

More general boundary conditions that include these as a subset can be used. We have used other and more general boundary conditions and no improvement in predicting the local velocity is achieved. Higher order terms greatly complicate the analytic formula and reduce 
the error in the formula by less than a few percent per term. Thus our extended solution only includes one higher order $\sin \theta$ term than does the Kuwabara solutiou. However as we shall show in Section 4 the above boundary conditions are adequately satisfied for many situations. Other expansion terms need to be included for the anisotropic unit cells and this will be discussed in Section 3.3.

The resulting extended solution for the total strearn function is

$$
\begin{array}{r}
\psi(r, \theta)=R\left[C_{1}\left(\frac{r}{R}\right)^{3}+C_{2} \frac{r}{R}+C_{3} \frac{R}{r}+C_{4} \frac{r}{R} \ln \left(\frac{r}{R}\right)\right] \sin \theta \\
+\left\{C_{5} R^{2} r^{3}\left[1+2\left(\frac{R}{r}\right)^{6}-3\left(\frac{R}{r}\right)^{4}\right]+C_{6} r^{5}\left[1+3\left(\frac{R}{r}\right)^{8}-4\left(\frac{R}{r}\right)^{6}\right]\right\} \sin (3 \theta),
\end{array}
$$

where $C_{5}$ and $C_{6}$ are given by

$$
\begin{aligned}
& C_{5}=-C_{7}\left(-8 R^{2}+256 R^{8}-384 R^{10}\right)-C_{8}\left(-R^{2}+256 R^{8}-768 R^{10}\right) \\
& C_{6}=-C_{7}\left(16 R^{4}-192 R^{8}+256 R^{10}\right)-C_{8}\left(4 R^{4}-192 R^{8}+512 R^{10}\right)
\end{aligned}
$$

where $C_{7}$ and $C_{8}$ are given by

$$
\begin{gathered}
C_{7}=\frac{4 C_{1}-16 R^{2}+16 C_{2} R^{2}+64 C_{3} R^{4}+16 C_{4} R^{2} \ln \left(\frac{1}{2 R}\right)}{C_{9}} \\
C_{8}=\frac{16 C_{1}-32 R^{2}+32 C_{2} R^{2}+64 C_{3} R^{4}+32 C_{4} R^{2} \ln \left(\frac{1}{\sqrt{2} R}\right)}{C_{9}}
\end{gathered}
$$

and $C_{9}$ is given by

$$
C_{9}=16 R^{6}-1344 R^{10}+6912 R^{12}-10572 R^{14}+8192 R^{18}
$$

The coefficients $C_{1}$ through $C_{4}$ are the coefficients of the Kuwabara solution and are given by Eqs. (17)-(20). The coefficients of this extended solution depend only on the porosity and the 
radius of the fiber and for a set of porosity and radius they are constants. The equations were manipulated using the Mathematica software ${ }^{1}$.

\subsection{Anisotropic Extension of Kuwabara Solution}

Many filters have fibers that are spaced closer together in the flow direction than in the perpendicular direction. To study the effect of this anisotropic distribution of fibers, we have examined anisotropic extension of the Kuwabara solution to an anisotropic array of fibers. More appropriately, this is an extension of the extended solution discussed in the last section.

As we will show this anisotropic extension to the Kuwabara solution does improve the accuracy of the solution for fibers spaced unequally in the $x$ and $y$ directions. However, the compactness of the coefficients found in the last section is lost.

The same geometry is used as in Figs. 1(b) and (c). However the periodic rectangular cells with sides $\ell_{x}$ and $\ell_{y}$ are used and the total area is still $\ell_{x} \ell_{y}=1$. Equation (2.4) from Sangani and Acrivos ${ }^{5}$ is no longer valid. It implicitly assumes the distance from the cylinder to the edge of the cell is equal in joth $x$ and $y$-directions. We find that one more extended term plus the one we added in the last section are needed to obtain reasonable solutions with at least some degree of compactness.

The method of solution is much the same as in Section 3.2, so we will only point out to the differences. The basic Kuwabara solution is not the most efficient zeroth order term, but with some small modifications it can be made into a first order term. We modify the Kuwabara solution by applying the boundary conditions on an ellipse with axes equal to the rectangular

\footnotetext{
${ }^{1}$ Mathematica is a Copyright of the Wolfram Research Inc.
} 
axes. We retain the extended term with undetermined coefficients of the last section. Finally we add a $\sin 2 \theta$ term that is the lowest order term and is not found in Eq. (24) because of the previously assumed symmetry. The same boundary condition is used as in the previous section only the integrals in Eqs. (36) and (37) are divided into smaller segments of the top boundaries.

The resulting solution is not as compact as Eq. (38). However, it becomes compact once a porosity and a ratio of $\ell_{y}$ to $\ell_{x}$ is chosen. The reason is the dependence of the coefficients on the porosity and the geometry is rather complex. For a porosity of 0.95 and a $\ell_{y} / \ell_{x}=1.44$, the solution is

$$
\begin{array}{r}
\psi(r, \theta)=9.362 \times 10^{-2}\left[\frac{7.714 \times 10^{-2}}{r}-4.723 r-7.809 r^{3}+9.943 r \ln (7.927 r)\right] \sin \theta \\
+\left[3.132 \times 10^{-1}\left(1+\frac{8.063 \times 10^{-6}}{r^{6}}-\frac{7.600 \times 10^{-4}}{r^{4}}\right) r^{4}-1.203 \times 10^{-1}\left(1+\frac{2.533 \times 10^{-4}}{r^{4}}\right.\right. \\
\left.\left.-\frac{3.183 \times 10^{-2}}{r^{2}}\right) r^{2}\right] \sin 2 \theta+\left[1.043 \times 10^{-1}\left(1+\frac{1.925 \times 10^{-7}}{r^{8}}-\frac{1.613 \times 10^{-5}}{r^{6}}\right) r^{5}\right. \\
\left.-2.252 \times 10^{-1}\left(1+\frac{8.063 \times 10^{-6}}{r^{6}}-\frac{7.600 \times 10^{-4}}{r^{4}}\right) r^{3}\right] \sin 3 \theta .
\end{array}
$$

The same anisotropy and a porosity of 0.80 gives the following expression

$$
\begin{array}{r}
\psi(r, \theta)=1.593 \times 10^{-1}\left[\frac{4.640 \times 10^{-1}}{r}-6.478 r-12.72 r^{3}+16.20 r \ln (3.963 r)\right] \sin \theta \\
+\left[1.172\left(1+\frac{5.160 \times 10^{-4}}{r^{6}}-\frac{1.216 \times 10^{-2}}{r^{4}}\right) r^{4}-5.625 \times 10^{-1}\left(1+\frac{4.053 \times 10^{-3}}{r^{4}}\right.\right. \\
\left.\left.-\frac{1.273 \times 10^{-1}}{r^{2}}\right) r^{2}\right] \sin 2 \theta+\left[2.374 \times 10^{-2}\left(1+\frac{4.928 \times 10^{-5}}{r^{8}}-\frac{1.032 \times 10^{-3}}{r^{6}}\right) r^{5}\right. \\
\left.-4.770 \times 10^{-1}\left(1+\frac{5.160 \times 10^{-4}}{r^{6}}-\frac{1.216 \times 10^{-2}}{r^{4}}\right) r^{3}\right] \sin 3 \theta .
\end{array}
$$

Equations (44) and (45) have been derived for an angle $\theta$ between 0 and $\pi / 2$. Using the same formula for angles larger than $\pi / 2$ leads to errors. For these angles symmetry is used to calculate the stream function. 
We have a closed-form analytic solutions for the coefficients of the above equation. These can be provided via e-mail (however, the length approximates the length of this article). These solutions can be manipulated via a computer algebra program such as Mathematica or Maple.

In Section 4 we show the results of this solution as compare them with the numerical results.

\section{Comparison of Various Solutions}

As previously discussed, numerical solutions to this problem are known (e.g., the numerical solutions of Sangani and Acrivos ${ }^{5}$ ) and are more accurate than the analytic solutions. Thus the numerical iesults from Section 2 will be our standard for the comparison.

The stream function contours using the Kuwabara, the extended, and the numerical solutions are shown in Fig. 3. Note that the Kuwabara solution in Fig. 3 (a) has streamlines crossing the upper boundary of the unit-cell in error. As we shall show later this has negative consequences for calculations of the particle collection efficiency. The extended analytic solution, Fig. 3 (b), and the numerical solution Fig. 3 (c), show that the flow does not cross the upper boundary of the cell as expected.

In Figs. 4 (a) and (b) we show the velocity errors in both the Kuwabara solution and the extended analytic solution. The velocity error fields for both are constructed by subtracting the velocity fields from that of the numerical solution. Both Fig. 4 (a) and (b) are scaled similarly. The magnitude of the largest error vector for the Kuwabara solution is 0.349 while that of the extended analytic solution is 0.119 and occurs at the inlet and exit boundaries of the unit cell. These results show that the extended analytic solution improves the prediction of the local flow field over the Kuwabara solution, especially near the upper boundary of the cell. This is also 
shown in Fig. 3 (b), where the stream function contours near the upper boundary is almost rectilinear, as expected. This is an important feature of the analytic solution since mass flow conservation is satisfied at every cross section of the square unit cell. The Kuwabara solution has a discontinuity in the upper boundary stream function when connected to the adjoint cell.

We have also made the same comparisons for $\epsilon=0.8$ and we have found that the discrepancy between the Kuwabara solution and the numerical solution is larger. The magnitude of the maximum error vector is 0.690 which occurs at the inlet and exit boundaries of the unit cell. The maximum error for the extended analytic solution is 0.205 .

The various solutions are also compared by evaluating the pressure drop for different porosities. The results are presented in Table 1 . These results show that for high porosity (i.e., $\epsilon \geq 0.8$ ) excellent agreement between the numerical solution and the analytic solutions is obtained. Note that for fibrous filters the porosity is larger than 0.8. For $\epsilon=0.7$, the difference between the extended analytic solution and the numerical solution is about $7 \%$ which is acceptable. For lower porosity (i.e., $\epsilon \leq 0.6$ ), the accuracy of the analytic solutions in predicting the pressure drop deteriorates further and a difference of $20 \%$ is obtained. Since the extended analytic solution is intended to correct the Kuwabara solution near the outer boundary of the unit-cell, the flow near the cylinder is not altered significantly. Thus, the Kuwabara and the extended analytic solutions predict pressure drops that are very close.

In Figure 5 we show the error for the extended anisotropic solution with $\ell_{y} / \ell_{x}=1.44$. The magnitude of the maximum error vector is 0.182 which is slightly larger than that for the square cell. Most of the error in the velocity vector occur near the exit boundaries of the unit cell. Thus for small stretches in the $y$-direction, the anisotropic analytic solution gives good estimates of 
the particle collection efficiency (this will be discusses below).

\section{$5 \quad$ Particles Impaction Efficiency}

To examine the utility of the extended solution, we begin with the particle impaction and the study of particle capture in filters with negligible diffusion, negligible surface forces, and for particles with negligible inertia. Without the inertial effects, the particle paths are entirely determined by the fluid flow. The opposite extreme is the case where the particle inertia is so large (i.e., the Stokes number is large) such that there are no fluid flow effects on the particle collection.

In Fig. 6, the geometry in aspects of the particle impaction are shown. A particle of radius $R_{p}$ is collected if it impacts the fiber, otherwise it is uncollected. There is a critical streamline which distinguishes between the collected and the uncollected particles for a given $R_{p}$. For these calculations, we assume that the fluid entering this unit cell has a uniform distribution of particles. The efficiency is simply the ratio of the collected particles to the total particles. For particles larger than the openings between fibers, sieving occurs and then they are all collected.

For each particle radius there is a limiting streamline that separates the flow region where particles are collected from the flow region where the particles are not collected. Thus the stream function with $\theta$ set to $\pi / 2$ and the radius $r$ replaced by the fiber radius $R$ plus the particle radius $R_{p}$ in Eq. (38), becomes an analytic relationship for the particle collection which is the advantage of having an analytic solution for the fluid flow. We can derive a particle collection formula rather than having to recalculate the efficiency for each particle. The particle capture 
efficiency is given by

$$
\begin{aligned}
\eta= & 200 \psi\left(R_{p}+R, \frac{\pi}{2}\right) \\
= & 200 R\left[C_{1}\left(\frac{R_{p}+R}{R}\right)^{3}+C_{2} \frac{R_{p}+R}{R}+C_{3} \frac{R}{R_{p}+R}+C_{4} \frac{R_{p}+R}{R} \ln \left(\frac{R_{p}+R}{R}\right)\right]- \\
& 200\left\{C_{5} R^{2}\left(R_{p}+R\right)^{3}\left[1+2\left(\frac{R}{R_{p}+R}\right)^{6}-3\left(\frac{R}{R_{p}+R}\right)^{4}\right]\right. \\
& \left.+C_{6} r^{5}\left[1+3\left(\frac{R}{R_{p}+R}\right)^{8}-4\left(\frac{R_{p}+R}{r}\right)^{6}\right]\right\}
\end{aligned}
$$

For small particle $\left(R_{p}<R\right)$, the atove efficiency expression can be simplified

$$
\begin{aligned}
\eta & =200 R\left[C_{1}+C_{2}+C_{3}+\frac{R_{p}}{R}\left(3 C_{1}+C_{2}-C_{3}\right)+C_{4} \frac{R_{p}}{R}\left(1+\frac{R_{p}}{R}\right)\right] \\
& -200\left(12 C_{5} \frac{R_{p}^{2}}{R^{3}}+24 C_{6} \frac{R_{p}^{2}}{R^{7}}\right)
\end{aligned}
$$

From Fig. 7 (a) and (b) one can see that close to the cylinder both the Kuwabara and the extended analytic solution are accurate. The extended analytic solution is also accurate from the cylinder to the edge of the periodic uait cell. The particle collection efficiency derived from the extended analytic solution is in excellent agreement with those from the numerical results. The results from the Kuwabara so'ution are less accurate than the extended analytic solution especially for lower porosity as shown in Fig. 7 (b). The Kuwabara error increases linearly while increasing the porosity. This is shown in Fig. 8 where the particle collection efficiency for $R_{p}=R / 10$ is shown for a variable porosity. For low porosity the discrepancy between the numerical and the Kuwabara solutions is large but the extended solution in in good agreement for the entire range of porosity shown.

Figures 9 (a) and (b) depict the efficiency predicted by the anisotropic extended solution given by Eqs. (44) and (45) and computed in the same manner as above for the square cell, 
i.e., Eq. (47). The results are for $\ell_{y} / \ell_{x}=1.44$. As shown, a good agreement is found with the numerical results for both porosities.

\section{Inertial Particle Collection Efficiency}

One major mechanism for collection of heavier particles in the absence of external forces, is the inertial impaction. In the previous section we ignored the particle inertia. The extent of the particle collection can be determined once the particle trajectories through the fiber lattice is known. The trajectories can be calculated by using the force balance on a particle. The Lagrangian particle momentum equation is given by ( $\operatorname{Tien}^{12}$ )

$$
c_{s} \frac{4}{3} \pi R_{p}^{*^{3}} \rho_{p}^{*} \frac{\mathrm{d} \mathbf{u}_{p}^{*}}{\mathrm{~d} t^{*}}=6 \pi \mu^{*} R_{p}^{*}\left(\mathbf{u}^{*}-\mathrm{u}_{p}^{*}\right)
$$

where $R_{p}^{*}$ is the radius of the particle, $u^{*}$ is the fluid velocity vector, $u_{p}^{*}$ is the particle velocity vector, $\mu^{*}$ is the dynamic viscosity of the fluid, and $c_{s}$ is the Cunningham correction factor which accounts for the velocity slip when the particle size is comparable to the mean free path of the fluid. The nondimensional form of Eq. (49) is

$$
S t \frac{\mathrm{d} \mathbf{u}_{p}}{\mathrm{~d} t}=\left(\mathbf{u}-\mathbf{u}_{p}\right)
$$

where $\mathrm{St}$ is the Stokes number defined by

$$
S t=c_{s} \frac{2}{9} \frac{\rho_{p}^{*}\left\langle u^{*}\right\rangle R_{p}^{*^{2}}}{\mu^{*} \ell^{*}}
$$

\subsection{Periodic Flow Regime}

For high Stokes numbers, the fluid flow has less influence on the particle trajectory and particle inertial effect dominates. For low Stokes numbers, the fluid flow dominates the particle 
trajectory. Thus the previous section on impaction is the limit for the small Stokes Numbers. Figure 10 shows some sample trajectories for $\mathrm{St}=0.1$ using the different solutions methods. The extended analytic solution predicts better the particle trajectories than the Kuwabara solution and both are compared with the numerical solution.

In Figs. 11 (a) and (b) we examine the particle collection efficiency for the Kuwabara, the extended and the numerical solutions. The results for two Stokes numbers are included. As in the previous section, we have assumed that the particles entering the unit cell are uniformly distributed in the fluid and have a local velocity equal to that of the fluid at the inlet boundary of the unit cell. The results of the previous section on the particle impaction, agree well with the small Stokes number results in Fig. 11(a).

As the Stokes number increases, the efficiency increases. In all cases the extended analytic solution gives a better prediction of the particle collection efficiency than does the Kuwabara solution. For small particle diameters, the Kuwabara solution and the extended solutions underpredict the particle collection efficiency. This is because the particle trajectories are strongly dependent on the initial conditions. Both the Kuwabara and the extended analytic solutions have a nonzero $v$-component of the velocity at the inlet of the unit cell. For the numerical solution, due to assumed symmetry, this velocity is very small compared to the $u$ component. The nonzero $v$-component of velocity in the analytic solution and for high Stokes numbers (i.e., St $>10$ ) makes the particle path oblique with respect to the $x$-axis of the unitcell. Hence the particle capture efficiency is lower than the numerical prediction. The effect of making the $v$-component of velocity zero at the inlet is shown in Fig. 12, for high Stokes numbers. The analytic solutions predict higher efficiency than the numerical solution. Note 
that for the numerical solution the trajectory of the particle is a straight line along the $x$-axis for high Stokes numbers, and therefore, the particle efficiency can be predicted from the geometry consideration and inlet velocity.

\subsection{Entrance Flow Regime}

In addition to the accuracy that the numerical method presents over the analytic solutions, it is able to simulate flow in the entrance to the filter. In this region the condition of periodicity of the fluid flow does not to apply. These numerical simulations show that for creeping flows the fluid flow becomes periodic beyond the first unit cell. The numerically obtained flow field is used to compute the particle collection for low and high Stokes numbers. For low Stokes numbers, the efficiencies are very close to those predicted using the flow field solution for the periodic (i.e., bulk). For high Stokes numbers, Fig. 13 shows that the efficiencies for the entrance region and the bulk region are different, because the particle trajectory is influenced by the initial conditions. For small particle diameters, the numerical results for the entrance region predict efficiencies which are closer to the analytic solutions. For large particles, the Kuwabara solution deviates, but the extended solution follows the same trend as the numerical results.

\section{Rectification of Particle Trajectories}

In the section on impaction, particles leaving one unit cell could not be collected in the next periodic cell unless some diffusion and/or a body force such as the gravity, an electrostatic or the van der Waals force are added. Since the limiting streamlines are periodic, then for all following cells in a periodic structure all particles on the dividing streamline (i.e., capturing streamwise 
mentioned in Section 5) barely miss collection on each downstream fiber past the first. The addition of an infinitesimal diffusion or a body force causes collection on these downstream fibers.

We propose that for the inertial impaction it may be possible for the in-line arrangement of fibers and downstream of the first fiber to have no collection of particles for a small but finite body force. To illustrate the phenomena, particle trajectory are given in Fig. 14 for the numerical, extended analytic and the Kuwabara solution. After the first fiber is missed, the particle gradually, after passing through a number of unit cells (fibers), moves toward the center point between the fibers. All trajectories of the particles moving parallel to the flow and not collected at the first fiber display this behavior.

To explain this behavior consider the extended analytic solution. To simplify the problem we will assume a large Stokes number. Thus to a first approximation, the trajectory is parallel to the $x$-axis. The fluid velocity in the Cartesian coordinates can be found from Eq. (25) by using the chain rule. To the first approximation, we assume that $y$ is nearly constant for large Stokes numbers and $v=0$ at the boundary. The nondimensional equation for the particle velocity is given by Eq. (50). To estimate the time it takes for the particle to transverse unit cell we can note that the fluid flow is symmetric about $x=0$. Thus the amount $U$ is retarded upstream it is accelerated by the fluid an equal amount down stream. This approximation is valid only as long as $y=$ constant is a reasonable approximation. Thus the time for crossing the unit cell is approximated by

$$
t=\langle u\rangle_{A} / x
$$


where $\langle u\rangle_{A}$ is the area average velccity along the $x$-direction is given by

$$
\langle u\rangle_{A}=\int_{-0.5}^{0.5} u(x, y) \mathrm{d} x
$$

To a first approximation we can assume $\mathrm{d} t=\mathrm{d} x /\langle u\rangle_{A}$. By use of the integration factor $\exp (t / S t)$ we can cast the $y$-component of the velocity as

$$
e^{t_{1} / S t} v_{p_{1}}-v_{p_{0}}=\int_{0}^{t_{1}} \frac{v}{S t} \mathrm{e}^{t / S t} \mathrm{~d} t
$$

Recasting the equation in $x$ rather than $t$ it becomes

$$
v_{p_{1}}=\frac{\mathrm{e}^{-x_{1} /\left(\langle u\rangle_{A} S t\right)}}{\langle u\rangle_{A}} \int_{0}^{x_{1} /\langle u\rangle_{A}} \frac{v}{S t} \mathrm{e}^{x /\left(\langle u\rangle_{A} S t\right)} \mathrm{d} x
$$

where we have substituted $t=x /\langle u\rangle_{A}$. We integrate once more to obtain an equation for $\delta y$, the displacement perpendicular to the $\mathrm{x}$-axis a trajectory receives as it passes through a unit cell is

$$
\delta y=y-y_{0}=\int_{0}^{1 /\left(2\langle u\rangle_{A}\right)} \frac{\mathrm{e}^{-x_{1} /\left(\langle u\rangle_{A} S t\right)}}{\langle u\rangle_{A}} \int_{0}^{x_{1} /\langle u\rangle_{A}} \frac{v}{S t} \mathrm{e}^{x /\left(\langle u\rangle_{A} S t\right)} \mathrm{d} x \mathrm{~d} x_{1}
$$

This formula can be anaytically integrated using the extended analytic solution and a symbolic algebra package such as Mathematica. The resulting formula is unwieldy. However, by breaking up the integration into two sections, before and aft of the the fiber, we can understand the effect analytically. From the above formula we can see that the positive $v$-component of velocity encountered before the cylinder displaces the near horizontal trajectory toward larger $y$ values. The velocities aft the fiber and in the opposite direction try to counter act the displacement that occurred upstream of the current fiber. The downstream fluid $v$-component of velocity are not able to counter act the full $y$ displacements resulting from the fluid upstream 
of the fiber. The reason that the positive $\delta y$ displacement occurring upstream of the fiber moves the trajectory up a bit and as it enters the downstream side the particle see smaller velocities because the particle is fari.zer from the fiber. Thus in each unit cell the particle receives a net displacement towards the center line between cylinders. Then there is a "rectification" of the particle displacement from the fluid flow. This effect occurs for any finite Stokes numbers. The Stokes number just determines how quickly this rectification occurs and over what number of cells it takes place. The final result is that after passing enough fiber particles move to the center line between fibers.

In other words, by integrating the velocity to obtain the small $y$ displacement we show that $\mathrm{d} y$ upstream of a fiber is positive and always greater than the smaller negative $\mathrm{d} y$ aft of the fiber. Since dy starts the downstream trajectory farther from the fiber, the $v$-component of velocities are less and the particle does not move to the original $y$ value. For large Stokes numbers, this $\mathrm{d} y$ is small, but accumulates for each fiber that is passed. The net effect is a rectification and after many cells the particle is moved away from the fiber and the possibility of collection is reduced even if small forces are included. Some sample $\delta y$ values are presented in Table 2 for different Stokes numbers. This process can be generalized for the in-line arrangement of fibers, beyond the doubly periodic arrangement considered here. This effect can be used to align particles.

\section{Conclusions}

The Kuwabara solution for flow through an in-line arrangement of cylinders has been extended and a compact extended solution is found. The extended analytic solution improves prediction for the efficiency of particle collection by fibrous filters. The advantage of this improved analytic 
solution is that it can be used for further analytic investigations of fluid flow in fibrous filters without undo complexity. The formula for the stream function is given by Eq. (38).

We calculate the analytic efficiencies for particle collection by impaction and show that the results agree well with the numerical simulations. We also investigate anisotropic arrangements of cylinders. The formulae for the stream function are given by Eqs. (44) and (45) using two porosities $\epsilon=0.95$ and 0.8 , respectively.

The "trajectory rectification", where the particles are less likely to be collected as they are moved away from the fiber by the fluid flow, is also discussed. A small body force would not alter this conclusion, because no collection past the first cell would occur beyond the first cell. However, a body force greater than a critical value determined by the Stokes number can alter this. Thus only diffusion and body forces larger than a critical magnitude would cause collection of particles beyond the first unit cell in the fiber lattice. In the case of negligible diffusion and body force, the fluid flow through these periodic structures can be used to align particles.

Acknowledgment: We would like to thank Dr. Werner Bergman of the Lawrence Livermore National Laboratory for his financial and technical support of this project. 


\section{References}

${ }^{1}$ S. Kuwabara, "The Forces Experienced by Randomly Distributed Parallel Circular Cylinders or Spheres in a Viscous Flow at Small Reynolds Numbers," J. Phys. Soc. Japan, 14, 727 (1959).

${ }^{2}$ D. O. Banks, "Stokes Flow Through a System of Parallel Infinite Cylinders with Axes Oriented at an Angle to the Direction of Mean Flow," Particulate Science and Technology, $5,339(1987)$.

${ }^{3}$ D. O. Banks, and G. J. Kurowski, "Inertial Efficiency of Cylindrical Collectors at an Angle to the Mean Flow Direction of Flow," Aerosol Science and Technology, 12, 312 (1990).

${ }^{4} \mathrm{C}-\mathrm{u}$ Choo and C. Tien, "Hydrosol Deposition in Fibrous Beds," Separations Technology, 1, $122(1991)$.

${ }^{5}$ A. S. Sangani and A. Acrivos, "Slow Flow Past Periodic Arrays with Application to Heat Transfer," Int. J. Multiphase Flow, 81, 193 (1982).

${ }^{6}$ M. Sahraoui and M. Kaviany, "Slip and No-Slip Velocity Boundary Conditions at Interface of Porous, Plain Media," Int. J. Heat Mass Transfer, 35, 927 (1992).

${ }^{\top}$ H. T. Prata and E. M. Sparrow, "Forced Convection Evaporation from a Cavity Containing a Liquid whose Surface is Curved by Capillarity: Computations in Interlocking Rectangular and Cylindrical Domains," Numer. Heat Transfer, 12, 667 (1985).

${ }^{8}$ S. V. Patankar, "Numerical Heat Transfer and Fluid Flow," (Hemisphere Pub. Co., Washington, DC, 1980).

${ }^{9}$ M. Kaviany, "Principles of Heat Transfer in Porous Media," (Springer-Verlag, New York, 1991). 
${ }^{10}$ B. Y. H. Liu and K. L. Rubow, "Air Filtration by Fibrous Filters," Fluid Filtration: Gas," 1, ASTM STP 975, Philadelphia, PA (1986).

${ }^{11} \mathrm{H}$. Hasimoto, "On the Periodic Fundamental Solution of the Stokes Equation and their Application to Viscous Flow Past a Cubic Array of Spheres," J. Fluid Mech., 5, 317 (1959). ${ }^{12} \mathrm{C}$. Tien, Granular Filtration of Aerosols and Hydrosols, (Butterworth Publishers, Stoneham, Massachusetts, 1989). 
Table 1 Pressure drop predictions for the Kuwabara solution, the extended, and the numerical solutions for the in-line arrangement of cylinders.

\begin{tabular}{cccc}
\hline$\epsilon$ & Kuwabara & extended & numerical \\
\hline 0.5 & 368.2 & 344.6 & 533.4 \\
0.6 & 184.1 & 177.9 & 218.3 \\
0.7 & 96.88 & 95.25 & 103.2 \\
0.8 & 49.94 & 49.64 & 50.26 \\
0.9 & 25.15 & 25.15 & 24.87 \\
0.95 & 15.74 & 15.76 & 15.57 \\
\hline
\end{tabular}


Table 2 Rectification of the particles trajectories crossing many cells shown by the vertical displacement of the particle at the boundary of each cell $(S t=0.1$ and $\epsilon=0.95)$.

\begin{tabular}{llll}
\hline Cell & \multicolumn{2}{c}{ Numerical Integration } & Analytic Integration \\
$\begin{array}{c}\text { Numerical } \\
\text { Velocity Field }\end{array}$ & $\begin{array}{c}\text { Extended } \\
\text { Velocity Field }\end{array}$ & \\
\hline 0 & 0.095 & 0.095 & 0.095 \\
1 & 0.140 & 0.151 & 0.156 \\
2 & 0.178 & 0.187 & 0.194 \\
3 & 0.211 & 0.218 & 0.226 \\
4 & 0.239 & 0.245 & 0.255 \\
5 & 0.262 & 0.268 & 0.274 \\
6 & 0.281 & 0.287 & 0.289 \\
\hline
\end{tabular}




\section{Figure Captions}

FIG. 1. (a) Periodic arrangement of cylinders with square unit-cell. (b) Boundary conditions used for the numerical solution. (c) Solution domain for the analytic solutions.

FIG. 2 (a) Unit-cell used to examine the effect of cell anisotropy on the pressure drop. (b) Pressure drop for creeping flow over an anisotropic, in-line arrangement of cylinders.

The pressure drop is normalized with the isotropic arrangement.

FIG. 3 Constant stream function contours for the (a) Kuwabara solution, (b) extended analytic solution, and (c) numerical solution $(\epsilon=0.95)$.

FIG. 4 Comparison of the local velocity vector between the two analytic solution and the two-dimensional numerical solution (a) extended analytic solution and (b) Kuwabara solution $(\epsilon=0.95)$.

FIG. 5 Comparison of the local velocity vector between the extended analytic solution for anisotropic unit-cell for $\epsilon=0.95$ and $\ell_{y} / \ell_{x}=1.44$

FIG. 6 Impaction Schematic.

FIG. 7 Particle impaction efficiency for uniformly distributed particles in the flow using (a) $\epsilon=0.95$ and $(b) \epsilon=0.5$.

FIG. 8 Collection efficiency error as a function of porosity.

FIG. 9 Particle impaction efficiency for ansotropic unit cells using uniformly distributed particles in the flow (a) $\epsilon=0.95$ and (b) $\epsilon=0.8\left(\ell_{y} / \ell_{x}=1.44\right)$.

FIG. 10 Sample trajectories for the different solutions.

FIG. 11 Particle collection efficiencies for (a) St=0.001 and (b) for St $=100$.

FIG. 12 Effects of initial conditions on particle collection efficiency using $v_{p}(-0.5, y)=0$ 
$(\mathrm{St}=100$ and $\epsilon=0.95)$.

FIG. 13 Particle collection efficiency at the entrance region of the packed bed of cylinders compared to the extended analytic solution, Kuwabara and the numerical solution in bulk for $\mathrm{St}=100(\epsilon=0.95)$.

FIG. 14 Particle displacement after crossing multiple cells. 


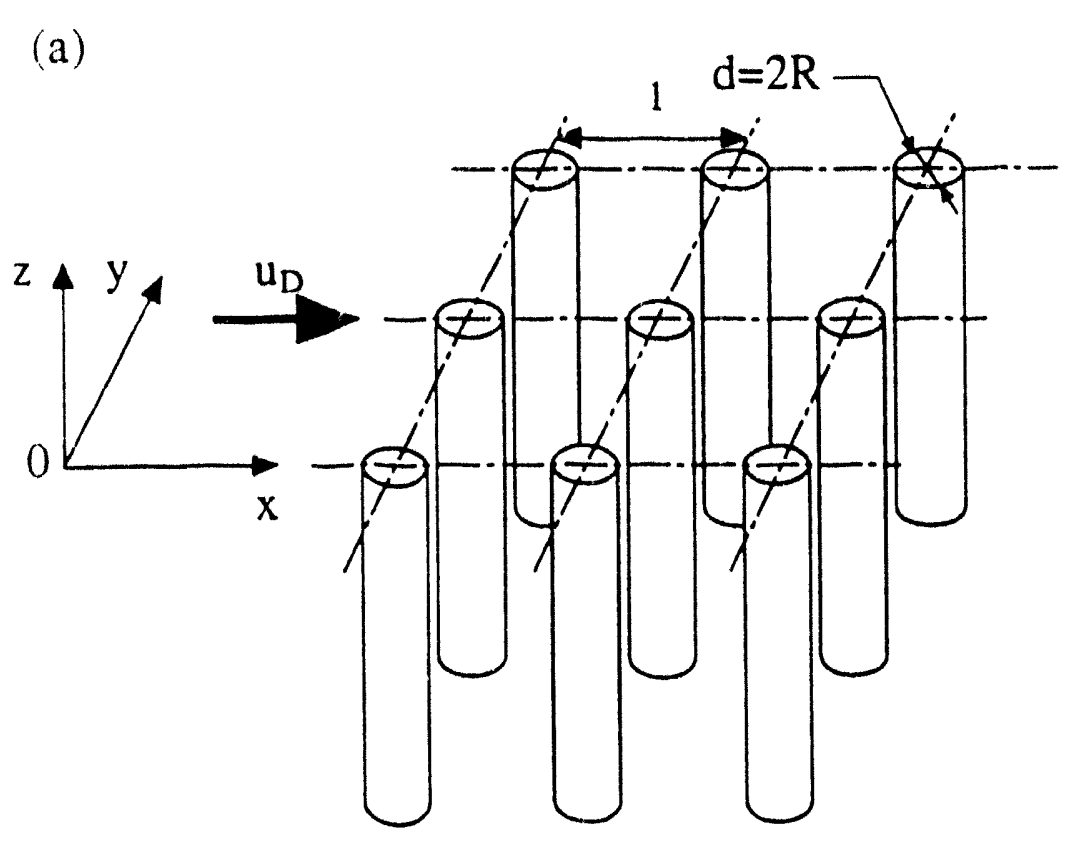

(c)

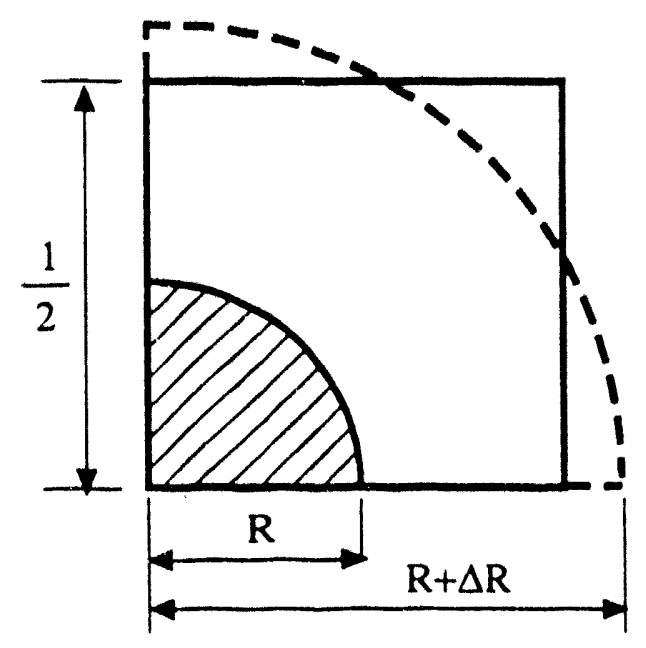

(b)

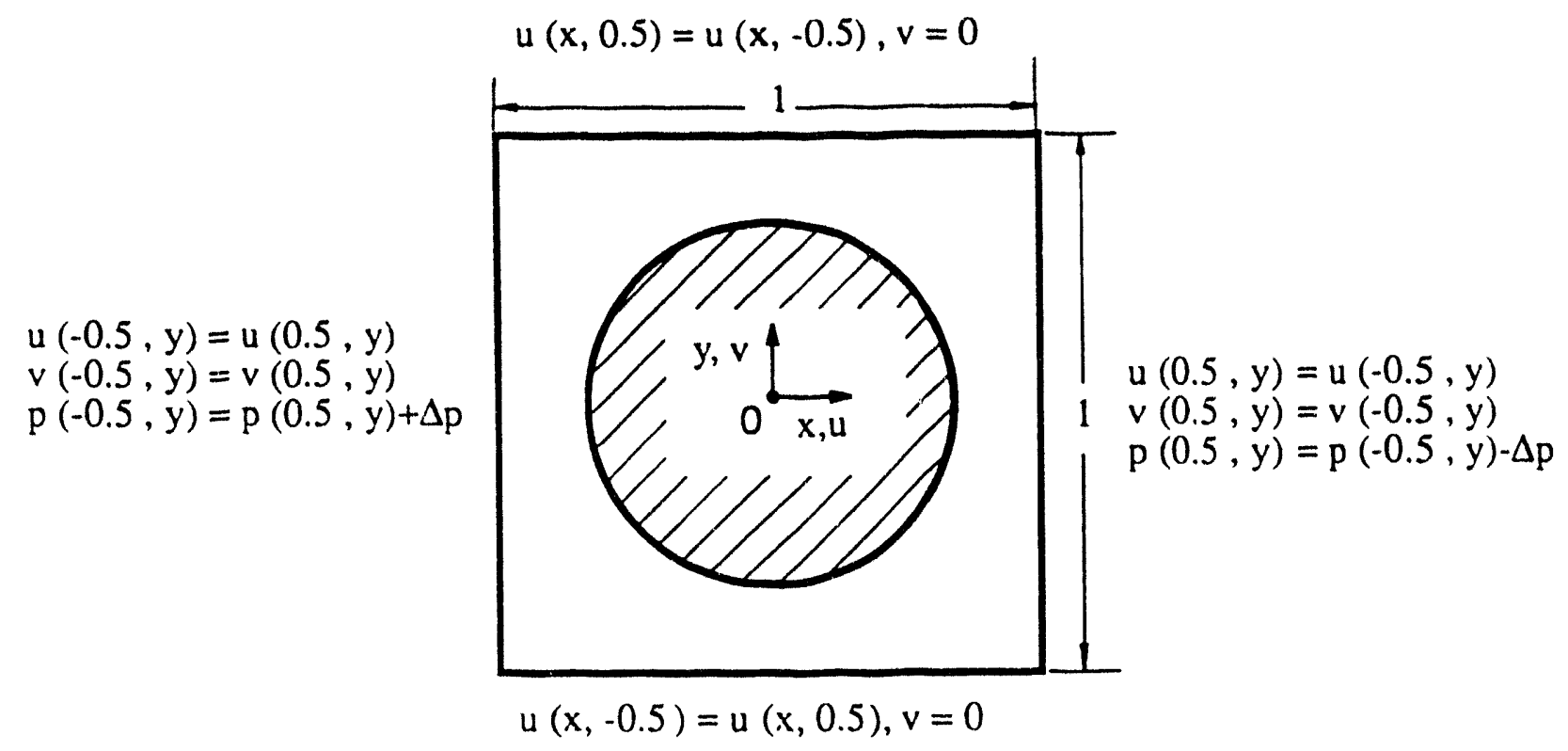

Figure 1 


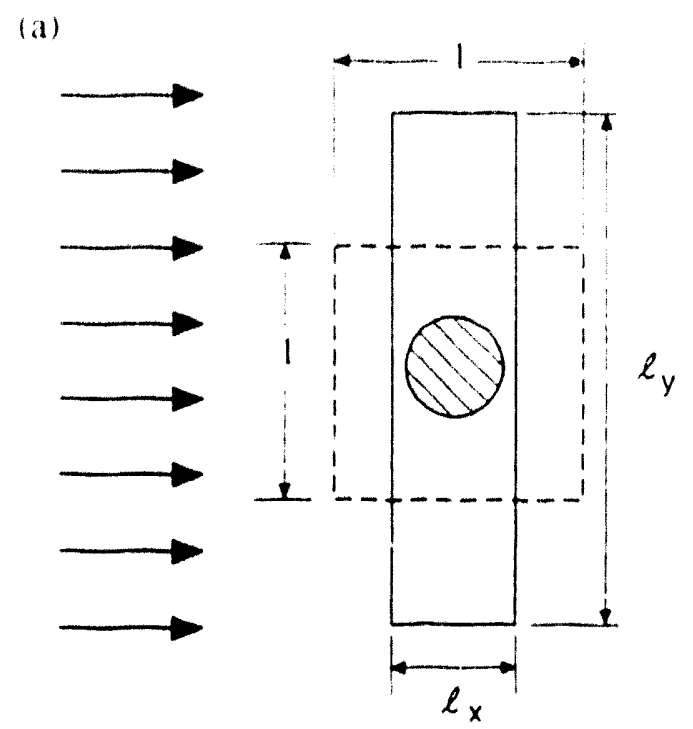

(b)

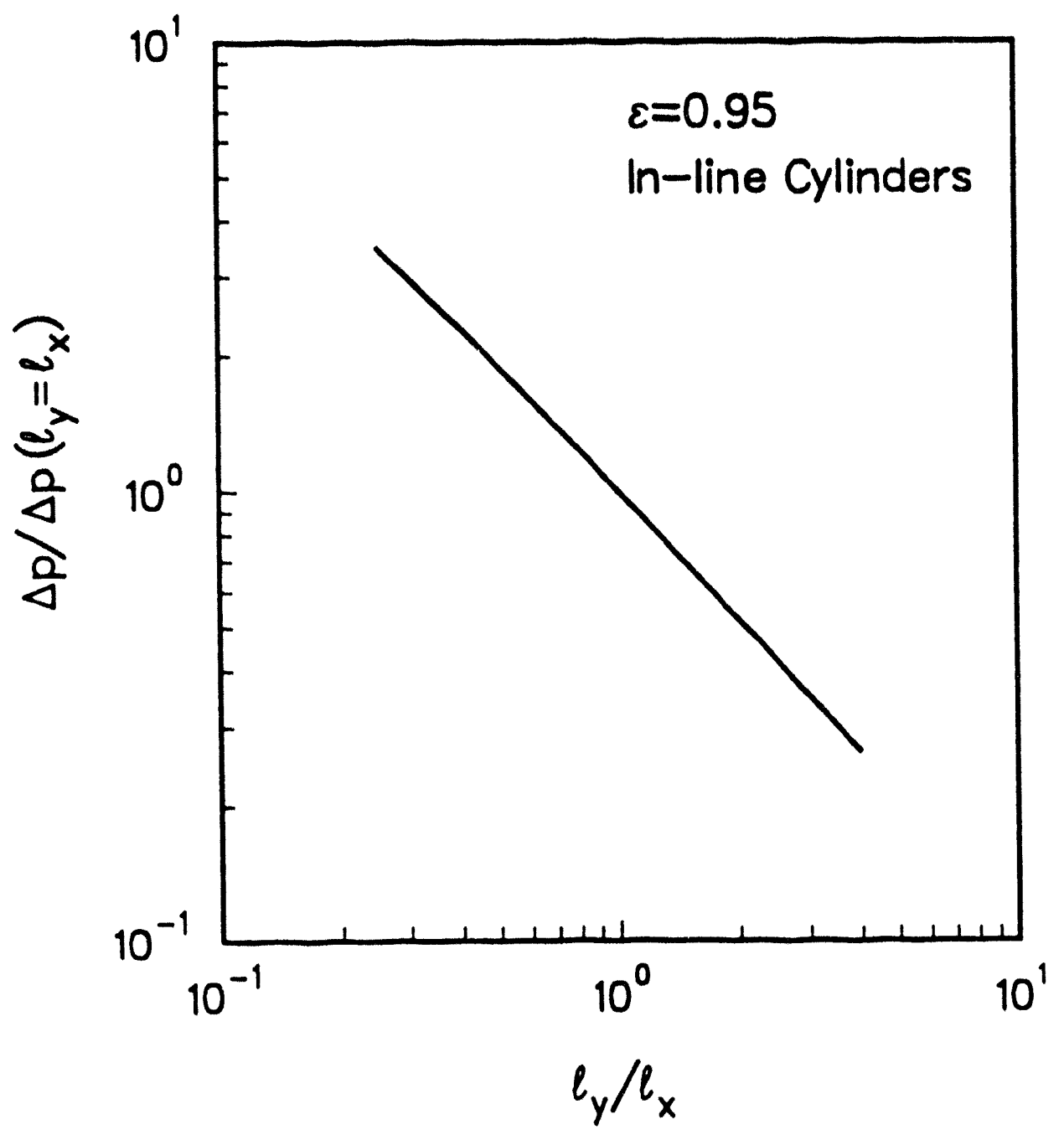

Figure 2 
(a)

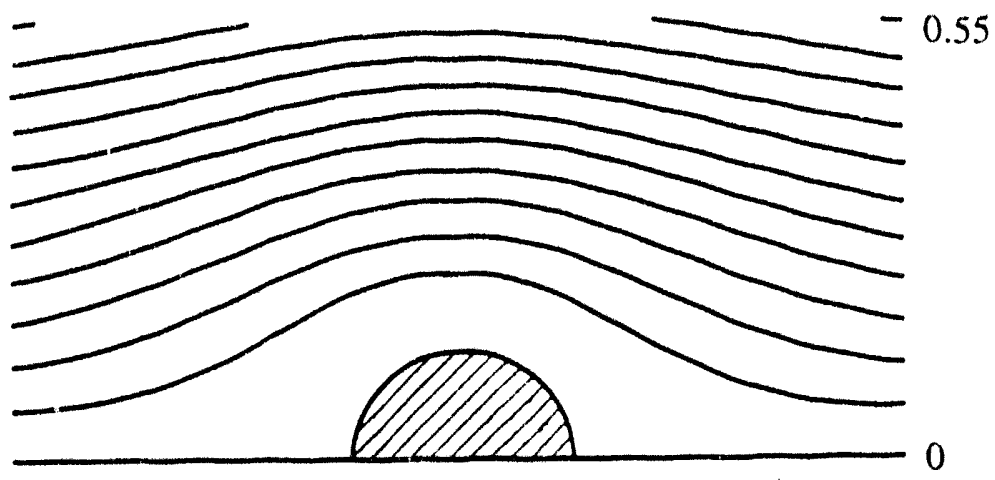

(b)

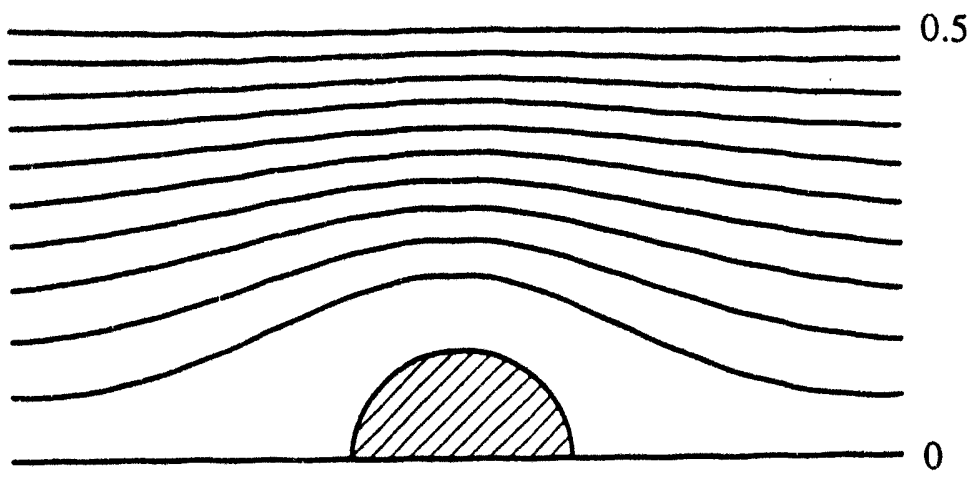

(c)

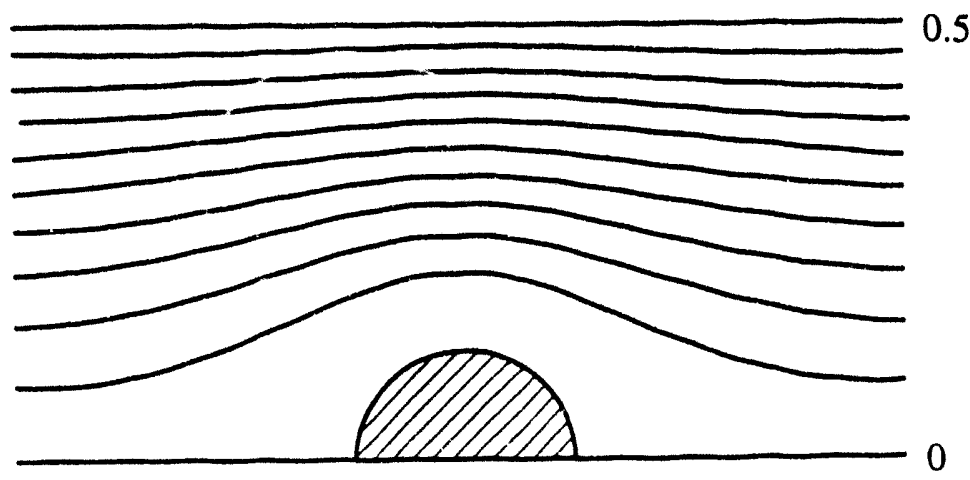

Figure 3 
† ว.กริ!

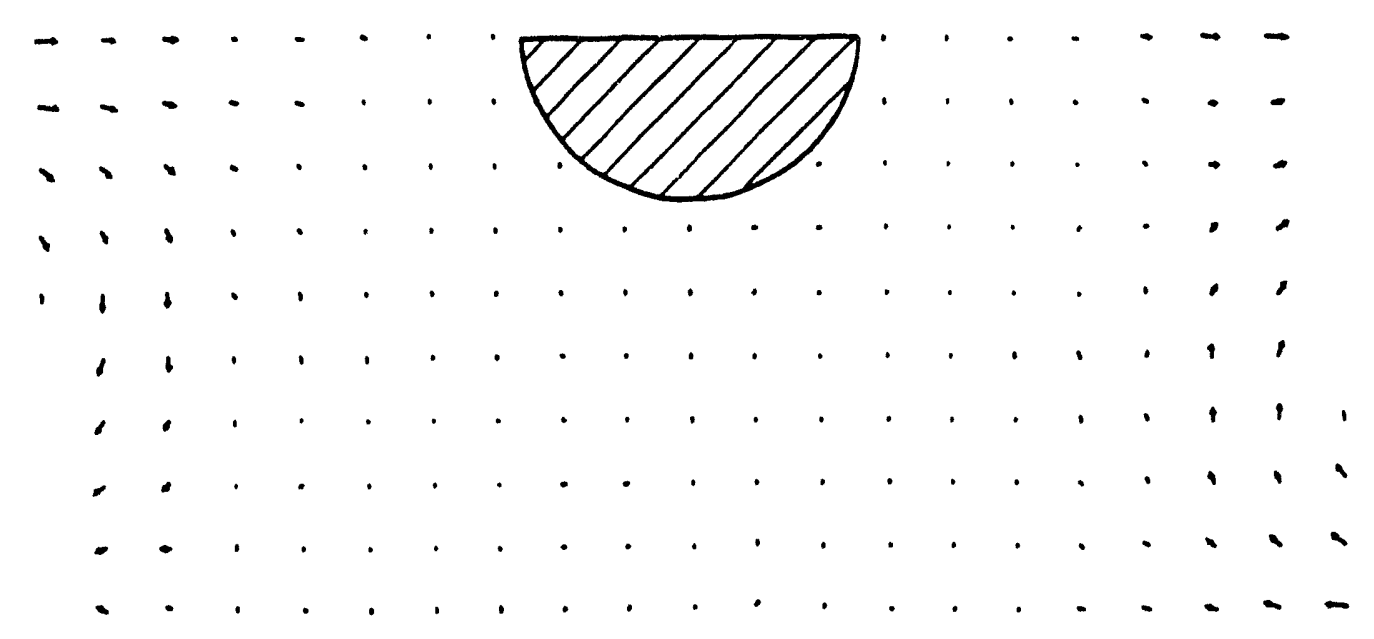

(9)

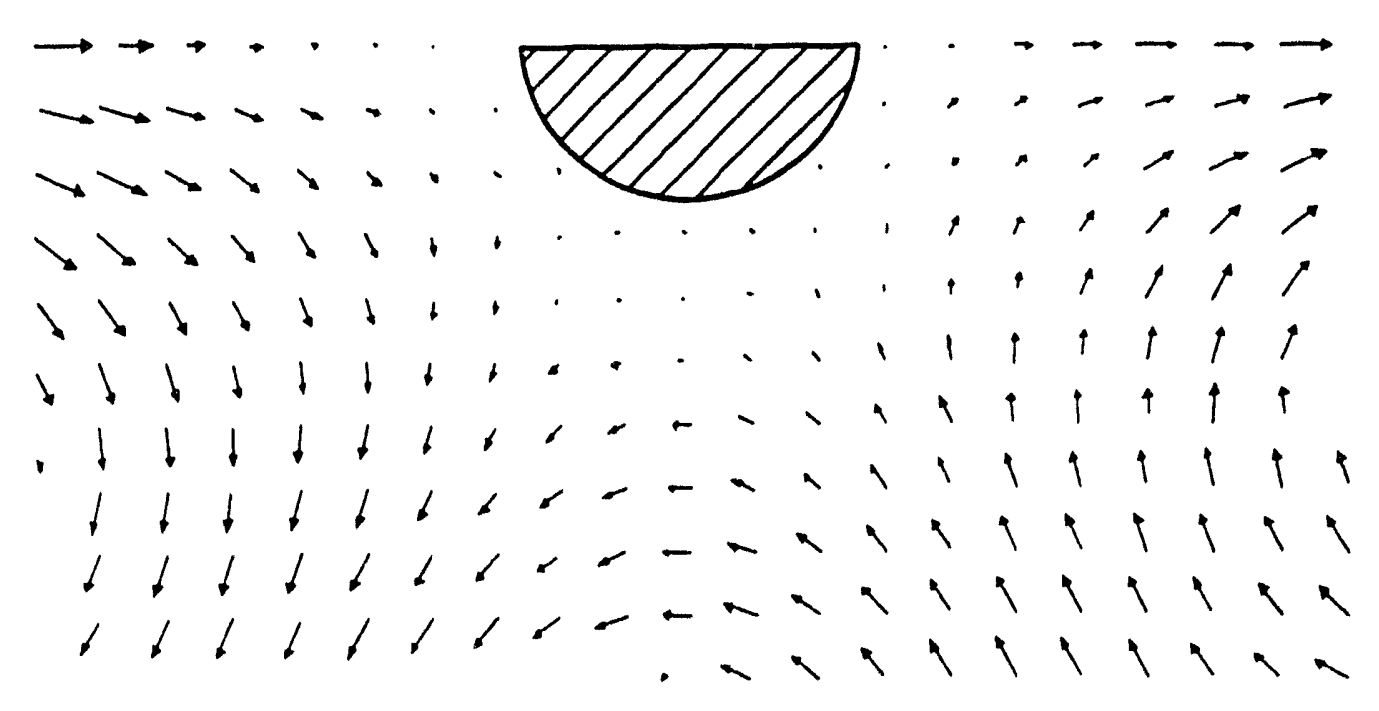


ऽ ว.กถิ!

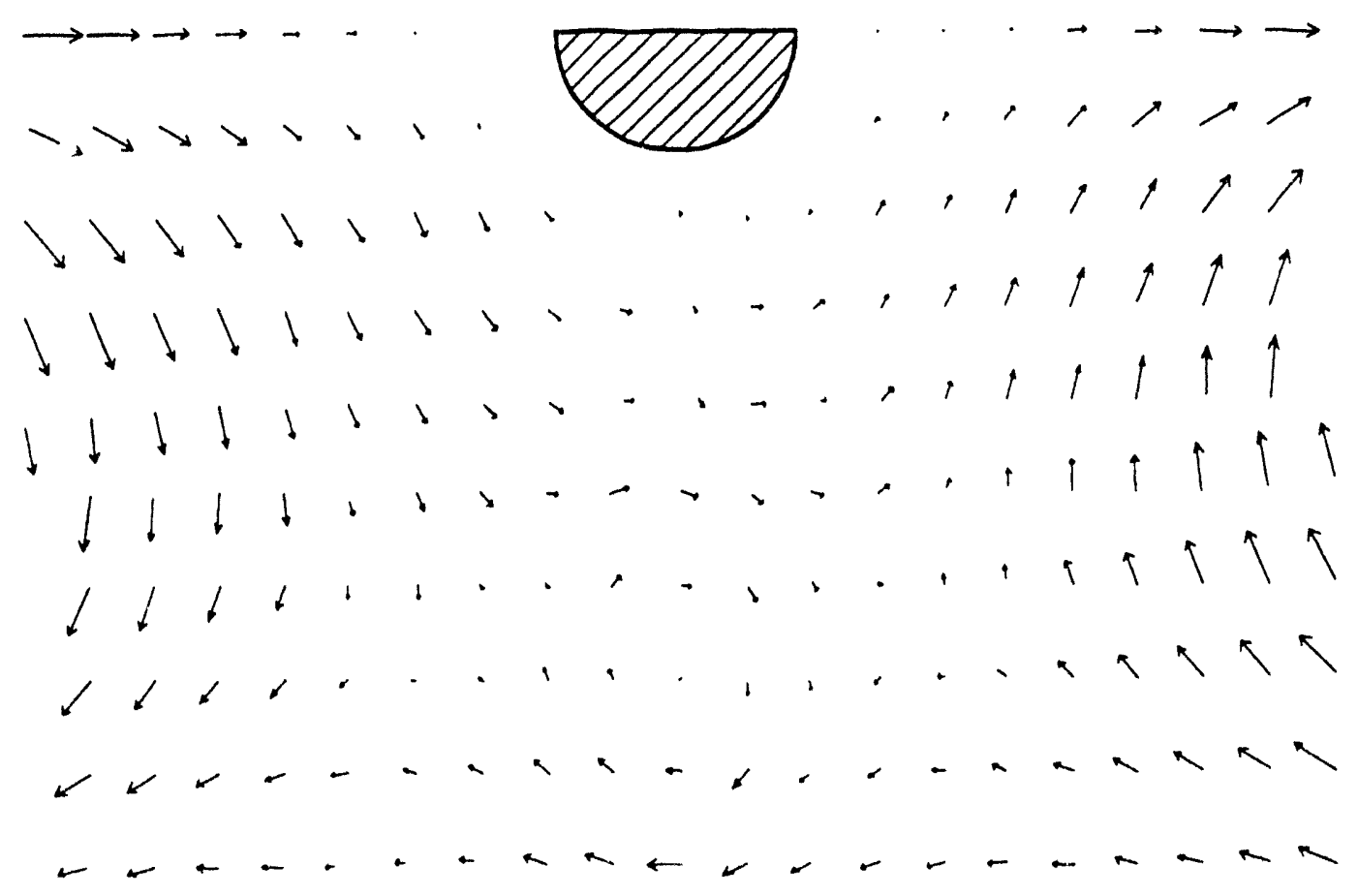




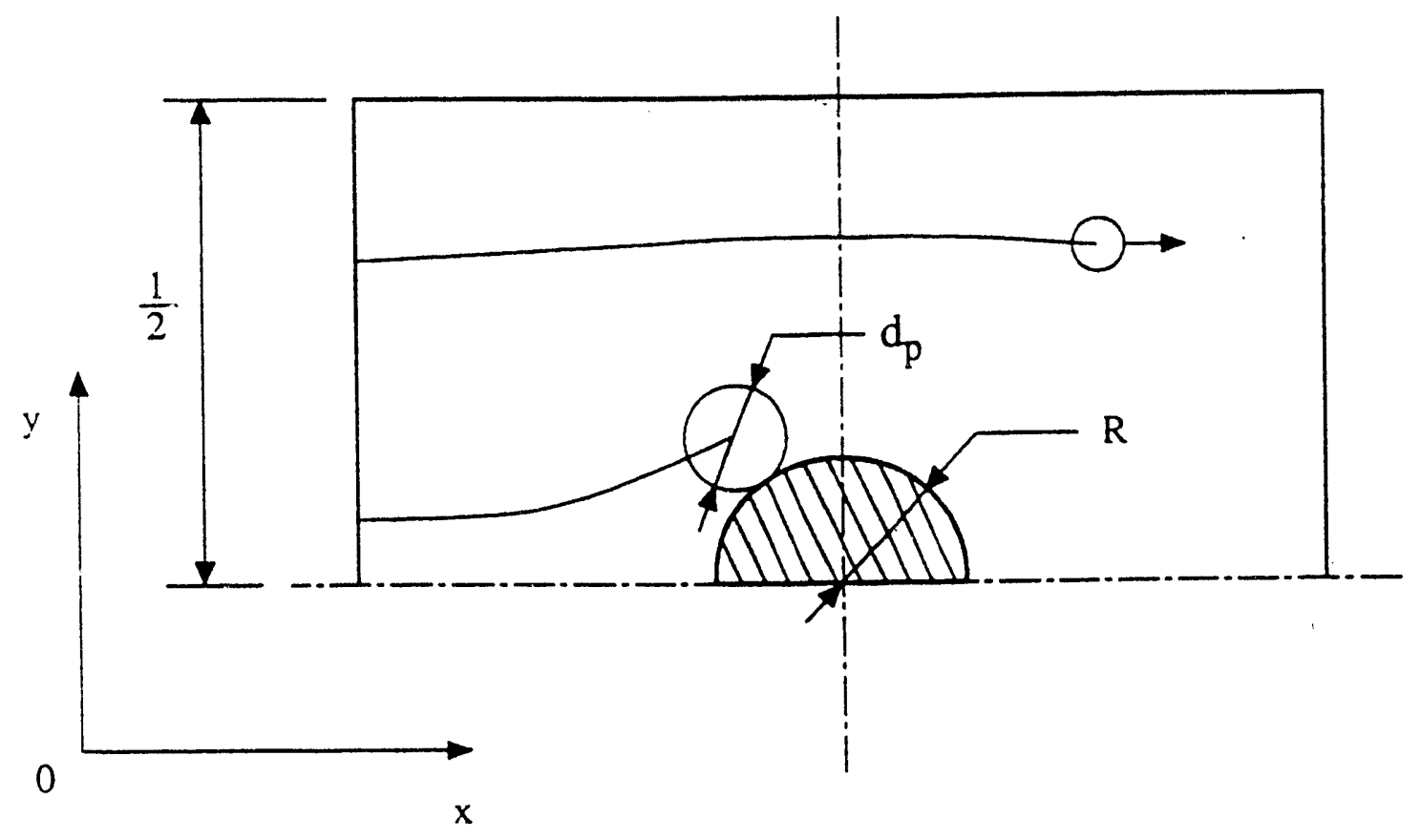

Figure 6 
(a)

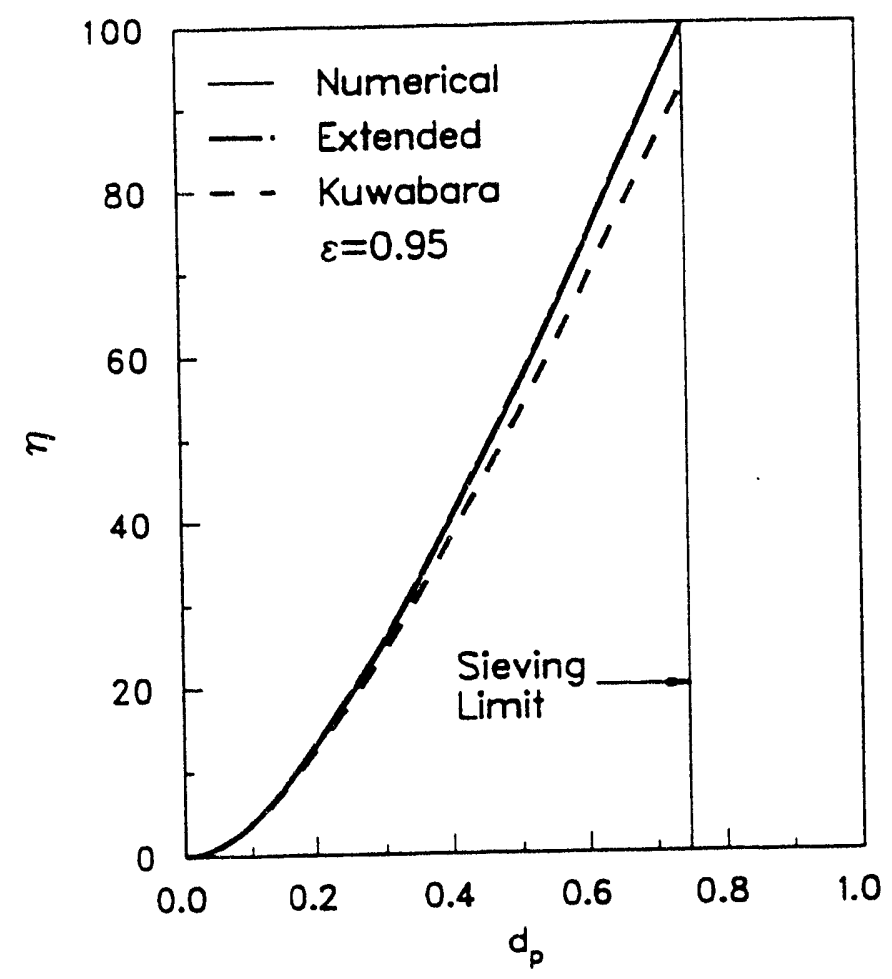

(b)

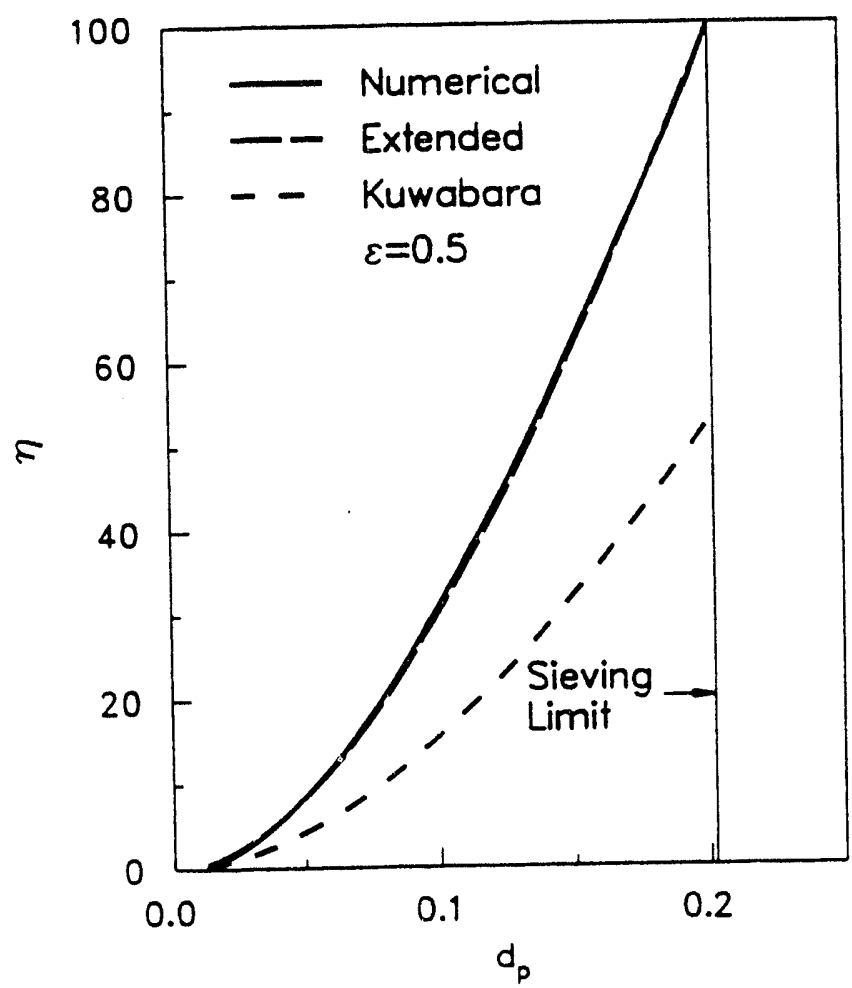

Figure 7 


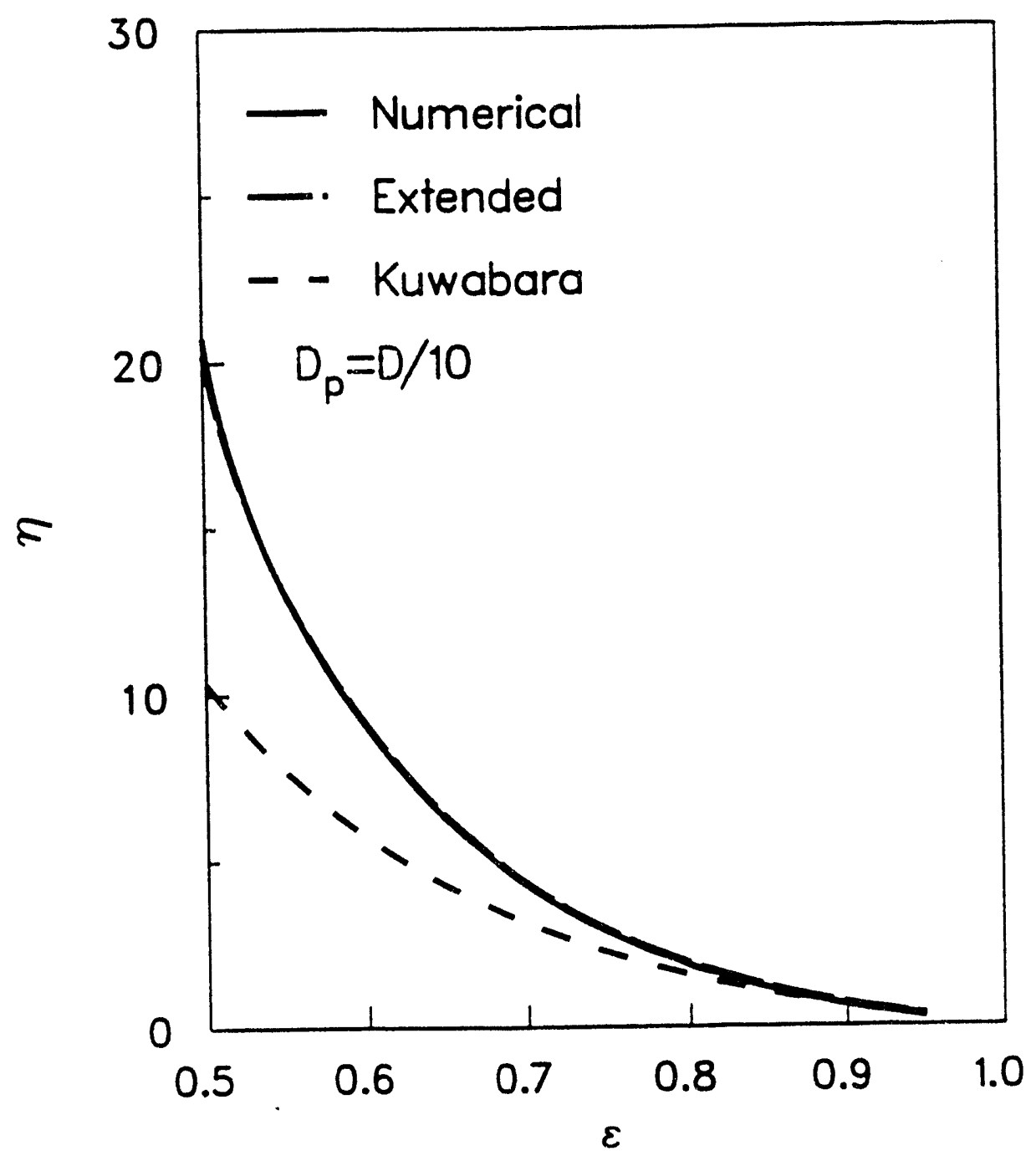

Figure 8 

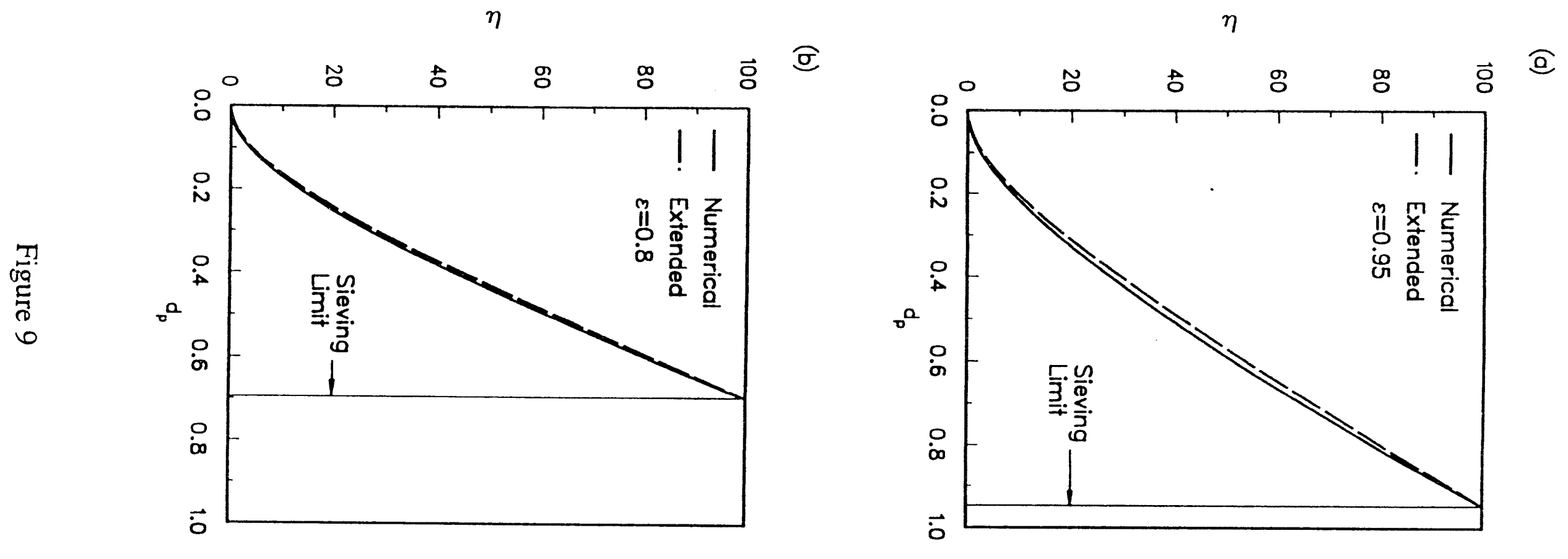
0I ว.กธิ!ี

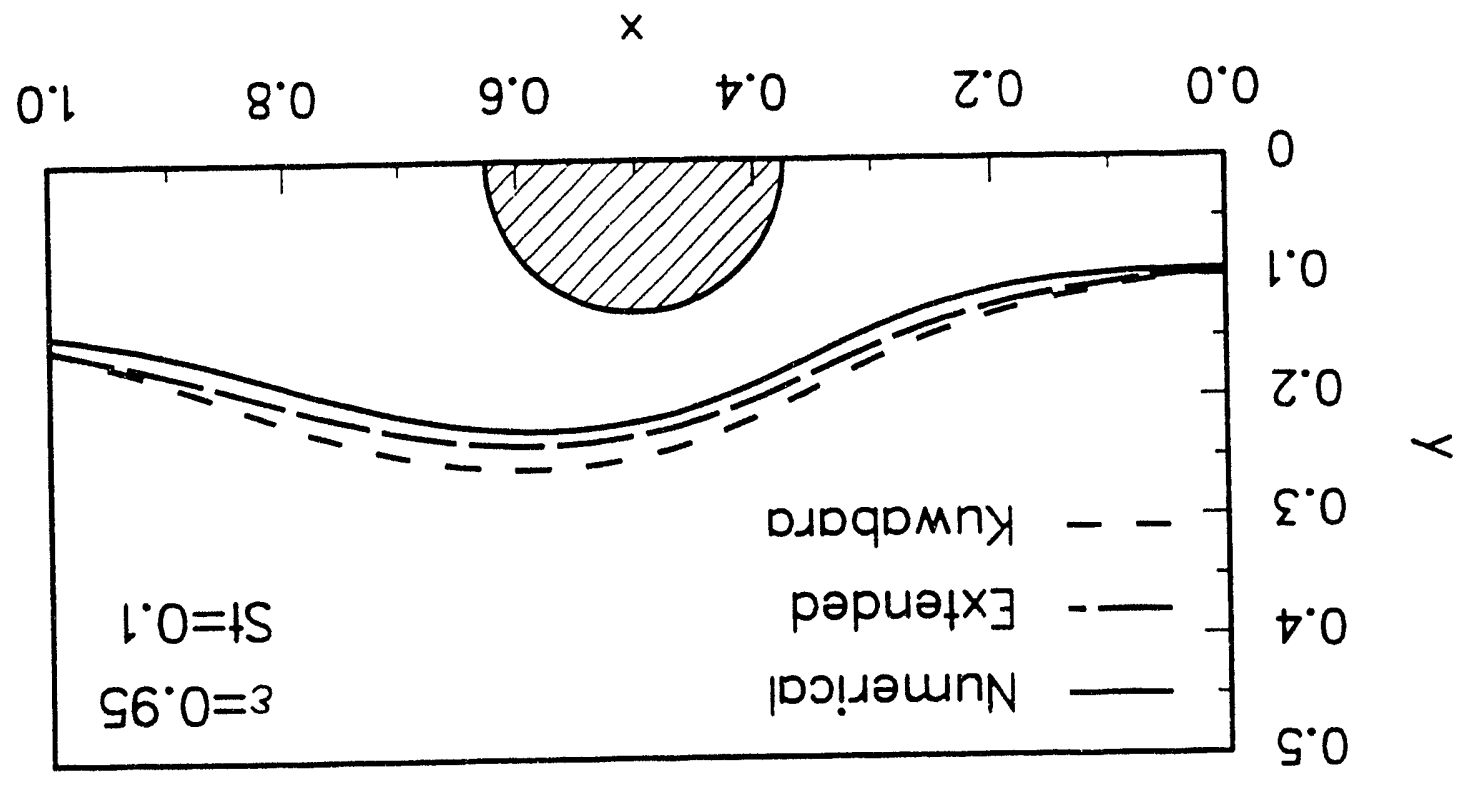


(a)

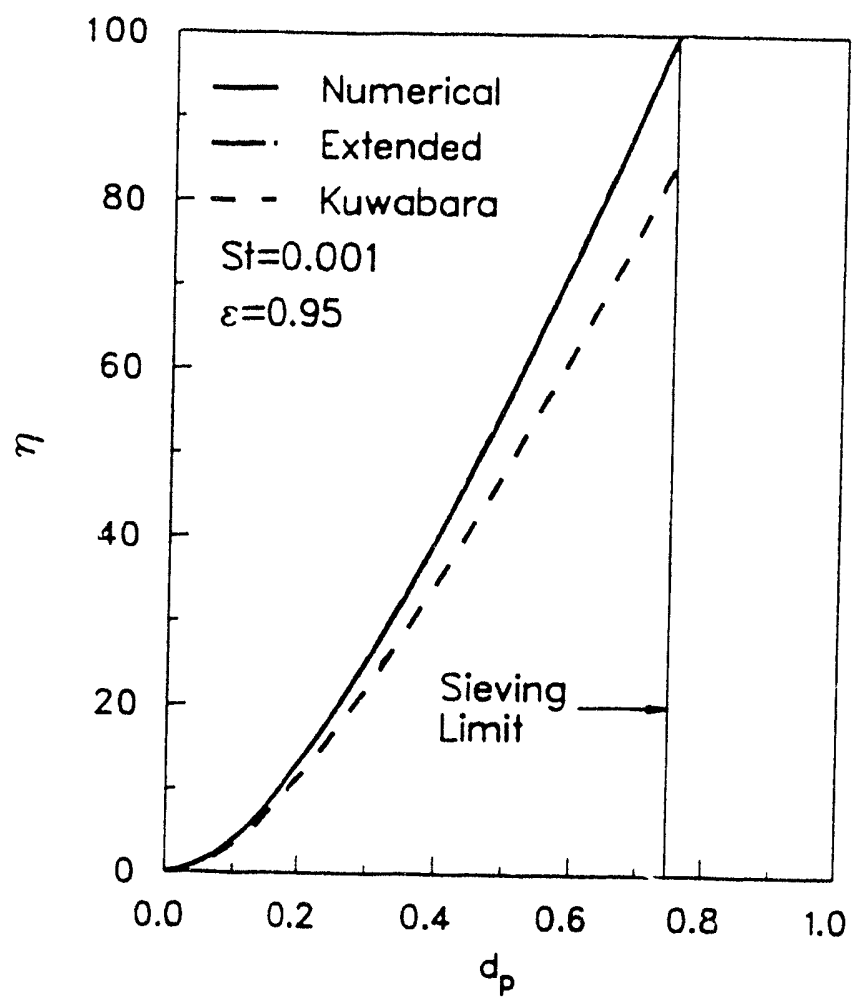

(b)

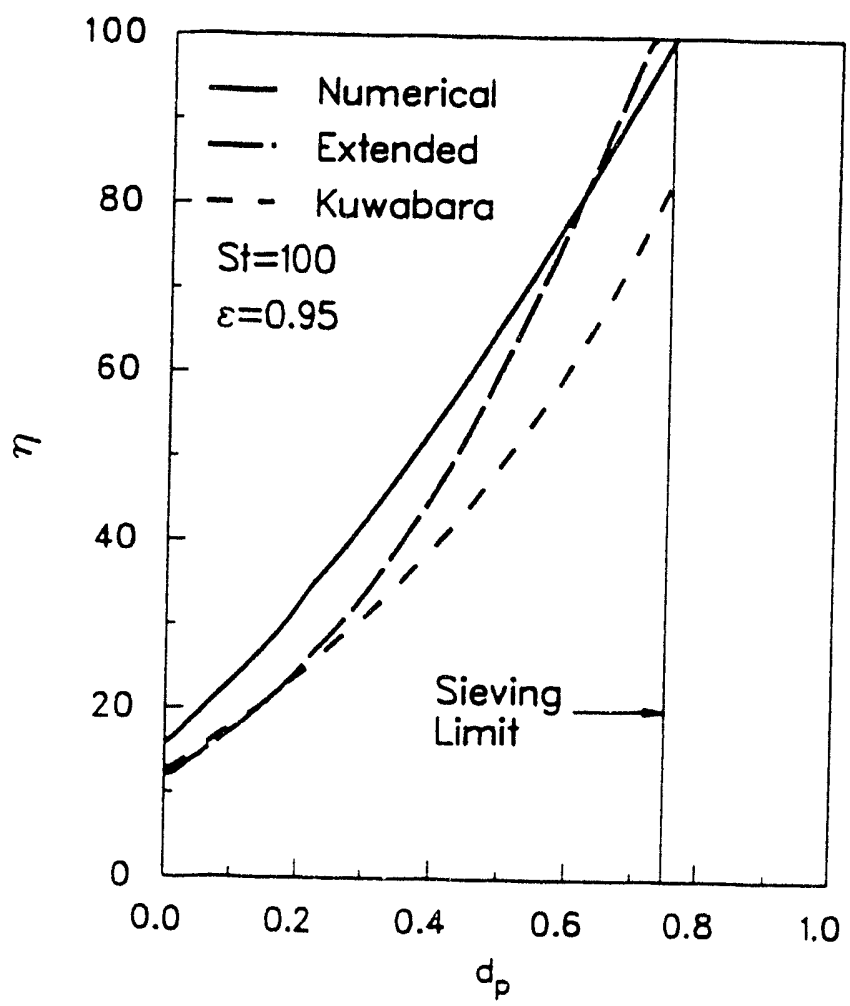

Figure 11 


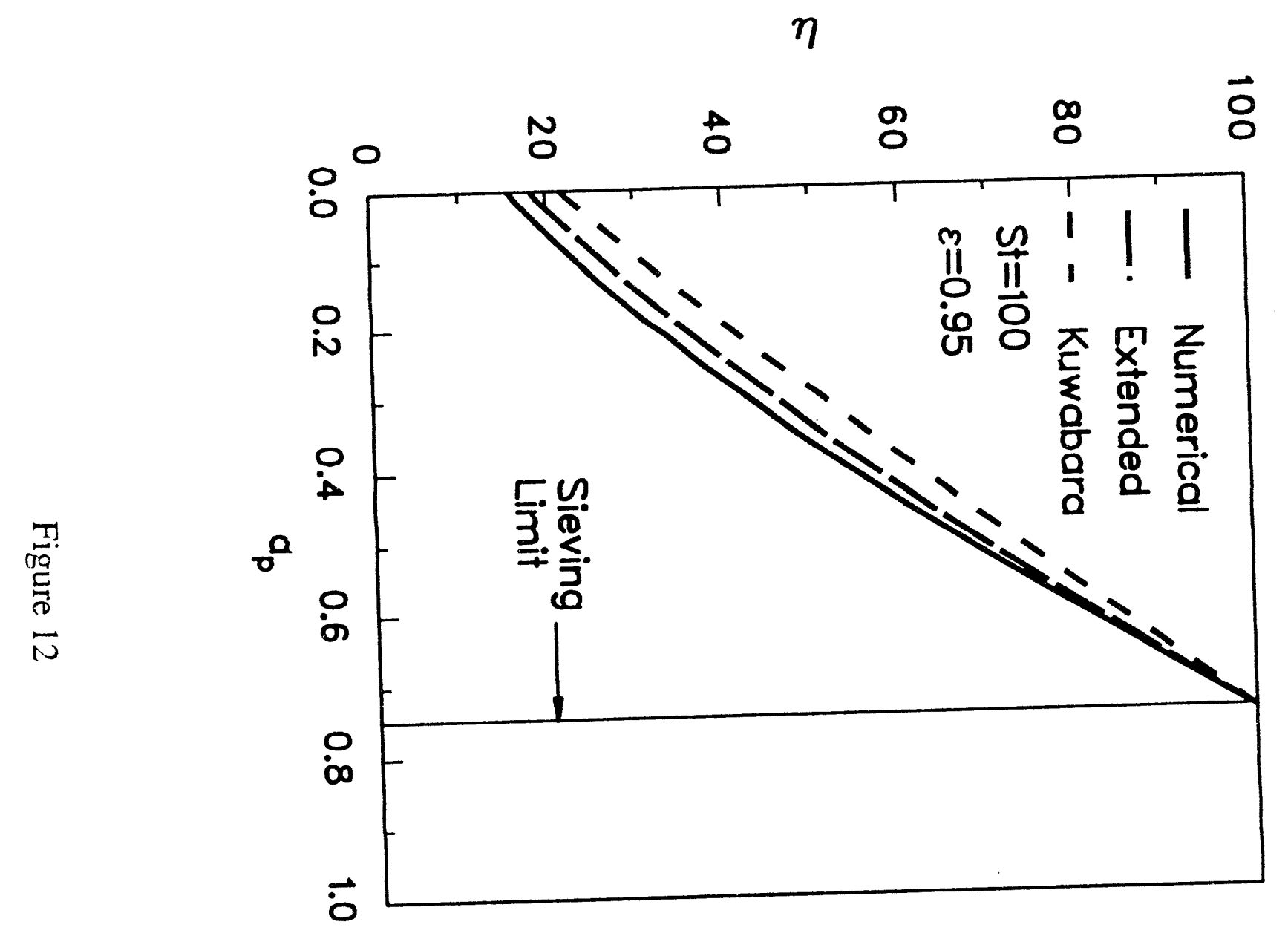




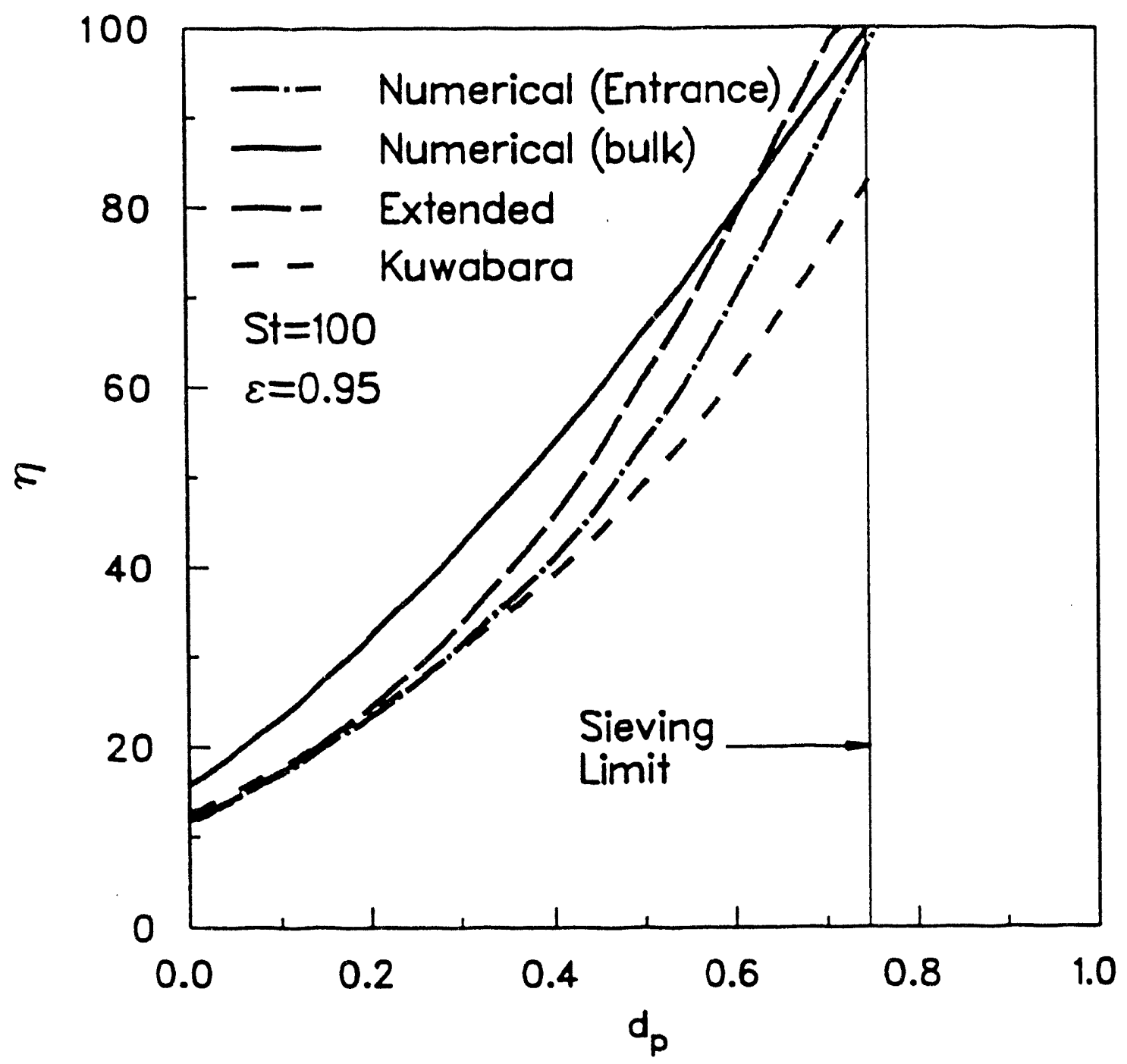

Figure 13 


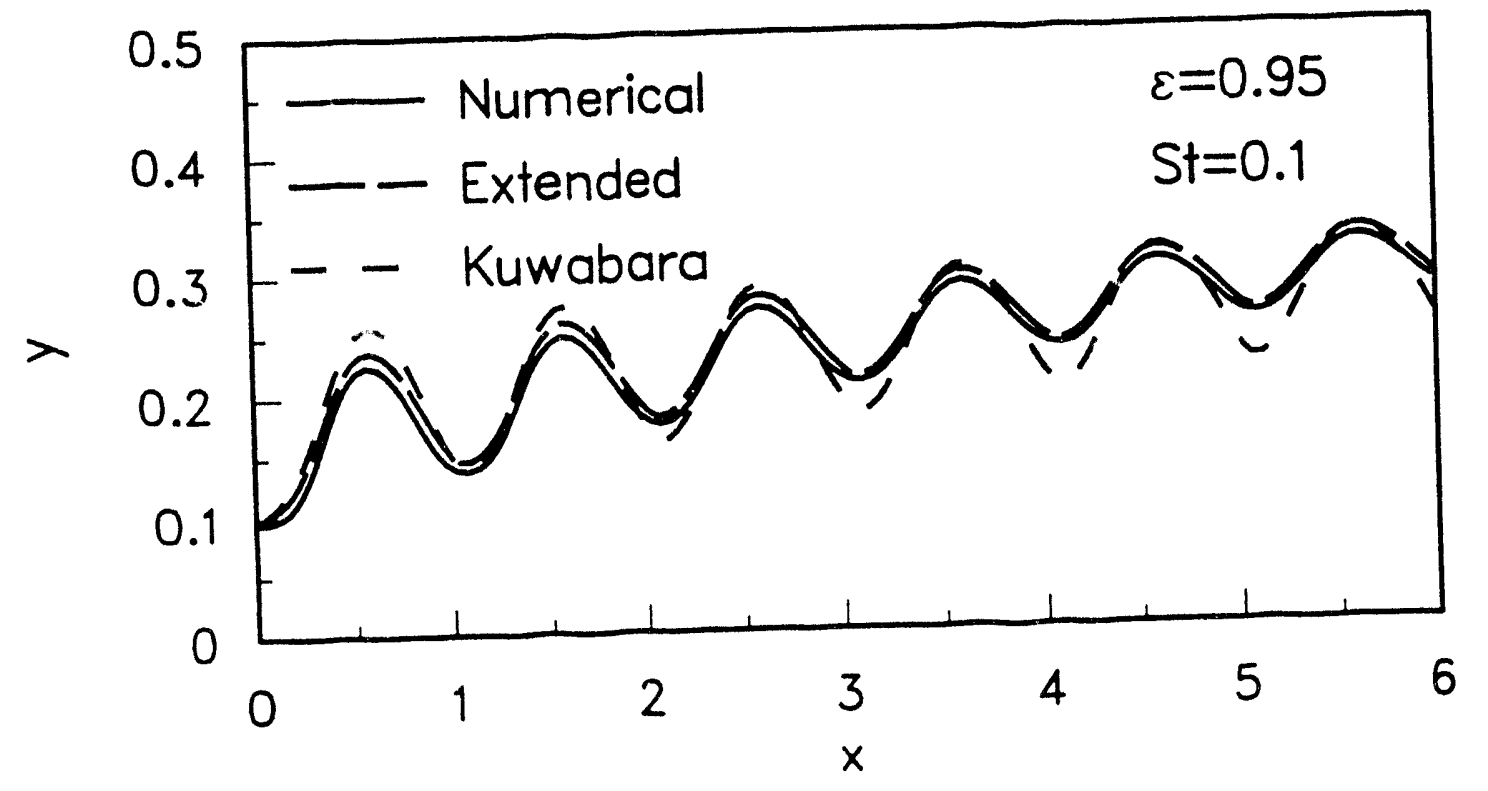

Figure 14 

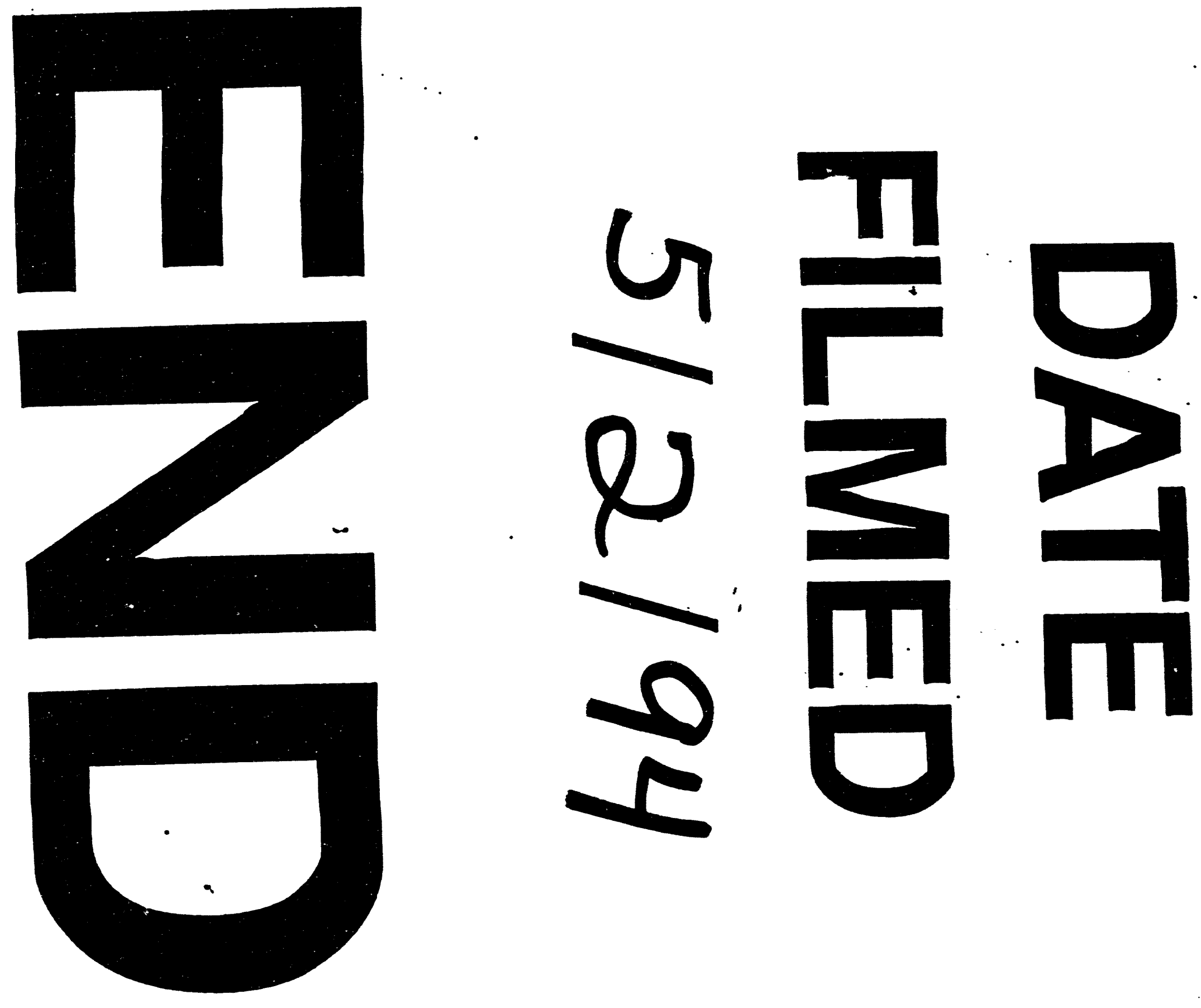
